\title{
Use of 3D-FEM Tools to Improve Loss Allocation in Three-Core Armored Cables
}

\author{
Juan Carlos del-Pino-López * (i) and Pedro Cruz-Romero
}

Citation: del-Pino-López, J.C.; Cruz-Romero, P. Use of 3D-FEM Tools to Improve Loss Allocation in Three-Core Armored Cables. Energies 2021, 14, 2434. https://doi.org/ 10.3390/en14092434

Academic Editor: Enrique Acha-Daza

Received: 15 March 2021

Accepted: 20 April 2021

Published: 24 April 2021

Publisher's Note: MDPI stays neutral with regard to jurisdictional claims in published maps and institutional affiliations.

Copyright: (C) 2021 by the authors. Licensee MDPI, Basel, Switzerland. This article is an open access article distributed under the terms and conditions of the Creative Commons Attribution (CC BY) license (https:/ / creativecommons.org/licenses/by/ $4.0 /)$.
Departamento de Ingeniería Eléctrica, Escuela Técnica Superior de Ingeniería, Avda. de los Descubrimientos s/n, Universidad de Sevilla, 41092 Sevilla, Spain; plcruz@us.es

* Correspondence: vaisat@us.es

\begin{abstract}
Loss allocation through analytical expressions in three-core lead-sheathed armored cables is challenging due to the complex geometry of this type of cable, commonly employed in submarine energy transmission systems (involving twisted conductors, sheaths and armor). Most of the expressions of the IEC standard 60287 have not been properly adapted for three-core armored cables, leading to inaccurate values for the different losses, so important efforts are currently devoted to improving them. In this work, an improved ultra-shortened 3D finite element model (FEM) is employed for developing an in-depth analysis of the electromagnetic interactions that take place in 6 real cables, being especially focused on those aspects that are not considered in the IEC standard. As a result, important conclusions are derived regarding the losses in conductors and sheaths, which introduce different corrections for improving the accuracy of the IEC expressions. The new formulation is then employed to propose a simplified experimental armor loss allocation procedure. This is virtually applied through the FEM tool to more than 700 cable configurations, showing a remarkable improvement in the loss allocation over the IEC standard and previous experimental procedures.
\end{abstract}

Keywords: three-core submarine cable; armor; sheath; losses; 3D FEM; conductor/armor twisting

\section{Introduction}

Loss allocation in power cables is a requirement for the thermal rating assessment that is performed at any stage of an underground/submarine high voltage transmission project (planning, design or operation). Due to the high number of offshore wind farms developed in recent years [1,2], the interest in this topic has grown significantly. In this sense, for three-core lead-sheathed armored cables the problem of loss allocation is even more challenging due to their three-heat source design (conductors, sheaths and armor, Figure 1). This was pointed out 10 years ago when [3,4] revealed that the IEC standard [5] greatly overestimates the power losses in this type of cable, hence obtaining oversized cables. The main reason is that [5] is partially based on studies developed in the 1930's, when cables had a different design. Moreover, some erroneous simplifying assumptions were included to obtain feasible analytical expressions (e.g., assuming the armor as a solid cylinder), leading to inaccurate values for the armor losses [3,4].

Thus, the problem of loss allocation in three-core armored cables (TCACs) has been tackled during the last 10 years, either in the form of new analytical formulations (for sheath and armor losses) [6,7], numerical methods, such as the method of moments (MoM-SO) [8], integral methods [9], the finite element method (2D FEM [3,4,10,11], 3D FEM [12-16]), multi-conductor cell [17-19] or more experimentally oriented [3,4,20-28].

Regarding the experimental methods $[26,28]$, even though they use real measurements of the global losses, they resort to [5] to allocate them. However, the oversimplified assumptions involved in the formulae lead to inaccurate values of the different loss components [16]. Hence, due to the difficulties of effectively separating the losses by experimental methods, 3D-FEM modelling is, without any doubt, the most accurate loss allocation method. However, due to the challenges in the FEM modelling of this type of cable (quite 
recently overcome $[14,16])$, and to avoid the need for using FEM analysis by non-specialized engineers, the use of straightforward analytical expressions is usually preferred.

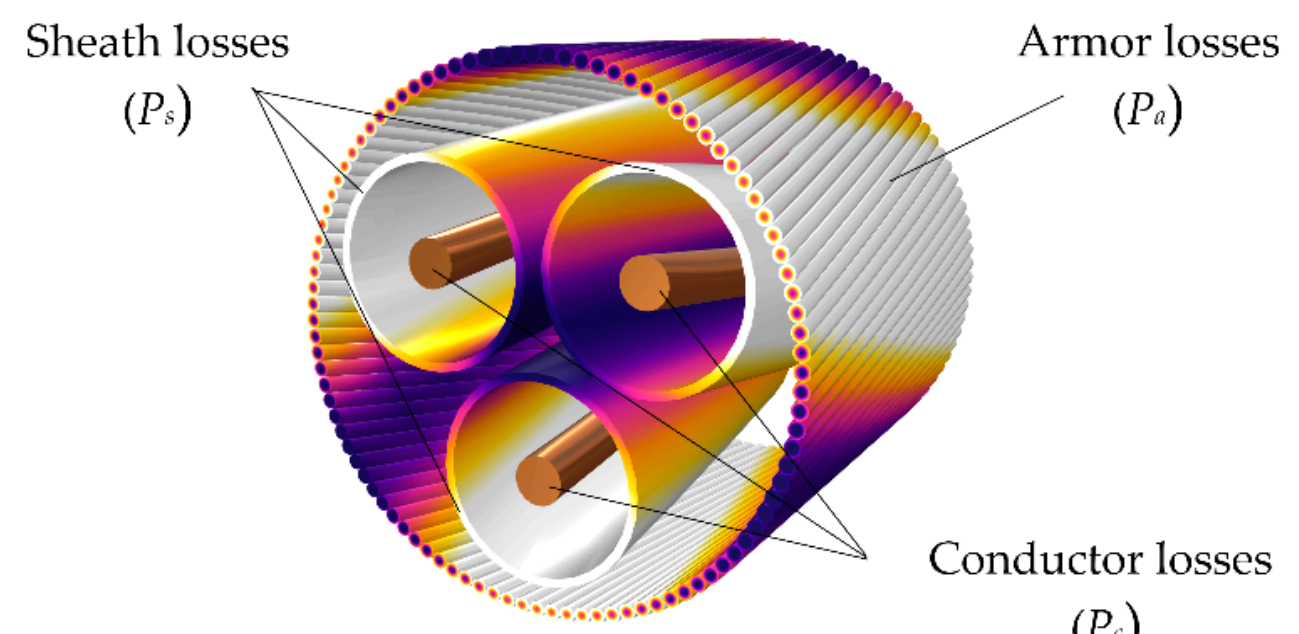

$\left(P_{c}\right)$

Figure 1. Example of loss color plot (white: high, purple: low) in a three-core armored cable.

In this sense, recent studies have suggested some modifications, as, for example, minor changes (e.g., increasing the additional length that every twisted phase conductor has compared to the overall axial length of the cable [29]), adding some empirically-based correction factors to include the effect of the armor in the conductor losses $[29,30]$ or, as cited before, developing new formulae for determining the sheath and armor losses [6,7,31]. However, the improvements obtained from most of these proposals are not properly reported and assessed in the literature.

Therefore, since the use of analytical expressions as those from [5] is preferred, advanced tools, such as 3D-FEM, are mainly devoted to help in the development of new and more accurate analytical expressions. To shed some light to the problem of loss allocation in TCACs, this work exploits the power of ultra-shortened 3D-FEM models to highlight those aspects where the formulae of [5] can be improved. This way, Section 2 briefly summarizes the traditional approach from [5], highlighting its main weaknesses. In Section 3 an ultra-shortened but accurate 3D-FEM model is proposed, including also new tools developed to get an in-depth knowledge of the physical phenomena involved. These tools are applied in Section 4 to analyze separately the conductor and sheath losses in 6 real cables. As a result, different corrections are proposed to enhance the accuracy of the formulation of these losses in [5]. Section 5 proposes a simplified experimental procedure that leverages these corrections to obtain a new assessment of the armor losses. Eventually, the conclusions are included in Section 6, also providing some remarks about open research lines aimed at obtaining eventually universal and accurate analytic expressions that could be incorporated in a future IEC standard version.

\section{Traditional Approach to Losses Estimation in TCACs}

In [5] the conductor losses $\left(P_{c}\right)$ are derived as follows:

$$
\begin{gathered}
P_{c}=3 R_{c}(\theta) I_{c^{\prime}}^{2} \\
R_{c}(\theta)=R_{c}^{D C}(\theta)\left(1+y_{s}+y_{p}\right), \\
R_{c}^{D C}(\theta)=\frac{\rho_{c}\left(\theta_{0}\right)}{S_{c}}\left(1+\alpha_{c}\left(\theta-\theta_{0}\right)\right),
\end{gathered}
$$

being $R_{c}(\theta)$ and $R_{c}^{D C}(\theta)$ the AC and DC conductor resistances at temperature $\theta$, respectively, $I_{c}$ the conductor current, $y_{s}$ the skin effect factor, $y_{p}$ the proximity effect factor, $\rho_{c}$ the 
conductor resistivity, $S_{c}$ the cross section of the conductor, $\alpha_{c}$ the temperature constant and $\theta_{0}$ the reference temperature. On the other hand, the sheath $\left(P_{s}\right)$ and armor $\left(P_{a}\right)$ losses are obtained by means of factors $\lambda_{1}$ (sheath loss factor) and $\lambda_{2}$ (armor loss factor), defined as

$$
\begin{aligned}
& \lambda_{1}=\frac{P_{s}}{P_{c}}, \\
& \lambda_{2}=\frac{P_{a}}{P_{c}} .
\end{aligned}
$$

Regarding sheath losses, [5] assumes that they are a consequence of eddy and circulating currents [32], so $\lambda_{1}$ can be defined as $\lambda_{1}=\lambda^{\prime}{ }_{1}+\lambda^{\prime \prime}{ }_{1}$, being $\lambda^{\prime}{ }_{1}$ and $\lambda^{\prime \prime}{ }_{1}$ the loss factors of circulating and eddy-current losses, respectively. However, [5] only provides expressions for $\lambda^{\prime}{ }_{1}$ and $\lambda^{\prime \prime}{ }_{1}$ in the case of three single-core cable arrangements, depending on whether the sheaths are in single-point bonding (SP) or solid bonding (SB) configuration. Hence, the same expressions have been applied for the case of TCACs, although only the corresponding to $\lambda^{\prime}{ }_{1}$ has been modified to include the effect of the armor (by introducing an empirical factor 1.5$)$ :

$$
\begin{gathered}
\lambda^{\prime}{ }_{1}=\frac{R_{s}(\theta)}{R_{c}(\theta)} \frac{1.5}{1+\left(\frac{R_{s}(\theta)}{X_{s}}\right)^{2}}, \\
\lambda^{\prime \prime}{ }_{1}=\frac{R_{s}(\theta)}{R_{c}(\theta)}\left[g_{s} \lambda_{0}\left(1+\Delta_{1}+\Delta_{2}\right)+\frac{\left(\beta_{1} t_{s}\right)^{4}}{12 \cdot 10^{12}}\right],
\end{gathered}
$$

with

$$
X_{s}=\frac{\mu_{0} \omega}{2 \pi} \ln \left(\frac{2 s}{d}\right),
$$

being $R_{s}$ the sheath resistance in $\Omega / \mathrm{m}, X_{s}$ the sheath reactance in $\Omega / \mathrm{m}, s$ the spacing between conductor axes, $d$ the mean diameter of the sheath, $t_{s}$ the sheath thickness, $\mu_{0}$ the vacuum permeability, $\omega$ the angular frequency and $g_{s}, \lambda_{0}, \Delta_{1}, \Delta_{2}$ and $\beta_{1}$ parameters defined in [5]. It should be remarked that in the case of sheaths in SB, [5] also assumes that $\lambda^{\prime \prime}{ }_{1} \ll \lambda^{\prime}{ }_{1}$, so it is neglected.

Nonetheless, there are some aspects of the design of this type of cable that are not adequately considered in this formulation. In this sense, previous works $[3,4,10,14,25]$ showed that phase and armor twisting, as well as the magnetic properties of the armor wires, increase the positive-sequence impedance of these cables. Therefore, $R_{c}, R_{s}$ and $X_{s}$ are affected by these aspects too. In this sense, [6-8] have suggested some modifications for improving the estimation of $R_{c}$. On one hand, considering the additional length of the conductors due to their twisting, leading to an increase in the conductor resistance [29], is suggested. This is done by multiplying $R_{c}^{D C}$ in Equation (3) by the lay factor $L F$, defined as

$$
L F=\sqrt{1+\frac{2 \pi R_{t}}{L_{c}}},
$$

where $L_{c}$ is the lay length of the conductors and $R_{t}$ denotes the distance cable centerconductor center. On the other hand, to include the influence of the armor on $R_{c}$ it has been suggested to multiply both skin and proximity factors in Equation (2) by 1.5 [30]. However, the source of this proposal is still to be published (technical brochure of CIGRE W.G. B1.56).

Although both improvements are directly applied to $R_{c}$, they also influence the sheath loss factor obtained by Equation (6), where $R_{c}$ plays an important role. In any case, the improvements derived from these proposals are not properly reported in the literature, so they must still be evaluated.

Another point of controversy is found in the bibliography regarding the source of the induced losses in the sheaths. Some studies $[6,7,33,34]$ suggest that eddy-current losses may be proportional to circulating-current losses for large cables, so they should not be 
ignored in the computation of the SB-case sheath losses. However, there is no expression in [5] especially designed for evaluating eddy losses in this type of cable (Equation (7) is in fact only valid for SP single-core cables). Hence, the effects of the core and armor twisting, as well as their magnetic properties, are not appropriately considered.

It is to be remarked that all these aspects cannot be accurately evaluated through laboratory measurements due to the impossibility of individually measuring the three loss components $[3,4,20,21,23-28]$, there existing still a degree of uncertainty that assigns erroneously the sheath eddy losses to other metallic elements. Nonetheless, regarding the armor losses, the experimental results are conclusive enough [3,4], verifying that [5] highly overestimates $\lambda_{2}$. In this sense, many efforts are now devoted to improving the accuracy of the expressions included in [5] (e.g., CIGRE W.G. B1.64 [31]).

As can be seen, although some improvements have been suggested in the formulation of the conductor losses, its accuracy still has to be properly evaluated. Moreover, the sheath and armor losses still require further improvements to a greater or lesser extent. All this is deeply analyzed in the following sections through the ultra-shortened 3D-FEM model presented next.

\section{Ultra-Shortened 3D-FEM Model}

In [14] it was proposed a new methodology to reduce the computational burden required for solving the 3D electromagnetic field in TCACs. For this task, rotated periodicity was introduced, based on the repetition of a certain electromagnetic field pattern shown by this type of cable in a length equal to the crossing pitch $(C P)$, a parameter defined in $[6,25]$ for cables with armor and phases twisted in opposite direction (contralay) as

$$
C P=\frac{1}{\frac{1}{L_{a}}+\frac{1}{L_{c}}}(\mathrm{~m}),
$$

where $L_{a}$ and $L_{c}$ are, respectively, the armor wire and phase conductor lay length. Thanks to this approach it is possible to analyze complex three-core cables with numerous wires in the armor by solving the electromagnetic field in just a short portion of the cable length equal to $C P$. Nonetheless, the simulation time may still be high for the largest cables, and it may be necessary to make use of a coarser mesh to reduce the number of degrees of freedom (DoF), thus solving faster, but providing less accurate results. To overcome this issue, new advances are presented in next subsections. The software Comsol Multiphysics [35] has been employed for this task.

\subsection{Ultra-Shortened 3D Electromagnetic FEM Model}

An in-depth analysis of the results obtained in numerous TCACs showed that the full electromagnetic behavior of the cable can be found in just a small slice of the cable, so a much shorter geometry for the FEM model can be employed [36,37]. This is possible since all armor wires are twisted around the three sheath-conductor cores, going through all the possible positions relative to every core, hence each one collects the full electromagnetic pattern. Therefore, it is possible to find a shorter section of the cable $(L)$ where the full electromagnetic pattern is distributed between all the armor wires included in that section. This length is

$$
L=\frac{C P}{n},
$$

where $n$ is any divisor of the number of armor wires $(N)$. An example is shown in Figure 2, where a $C P$-length section of a $115 \mathrm{kV}, 240 \mathrm{~mm}^{2}$ three-core cable is represented (for a clearer figure the loss color plot is only depicted for one armor wire). Considering that $N=98$ in this cable, the possible values for $n$ are 1, 7, 14, 49 and 98. In the case of Figure $2(n=7)$ it can be easily observed how the loss pattern shown by the selected armor wire is split into $C P$ /7-length sections, each one having the same loss distribution as other armor wires located every $N / 7$ th position in Section 1 of the figure. This way, the full pattern of that 
armor wire can be fully reproduced by adequately aligning those $C P$ /7-length armor wires of Section 1.

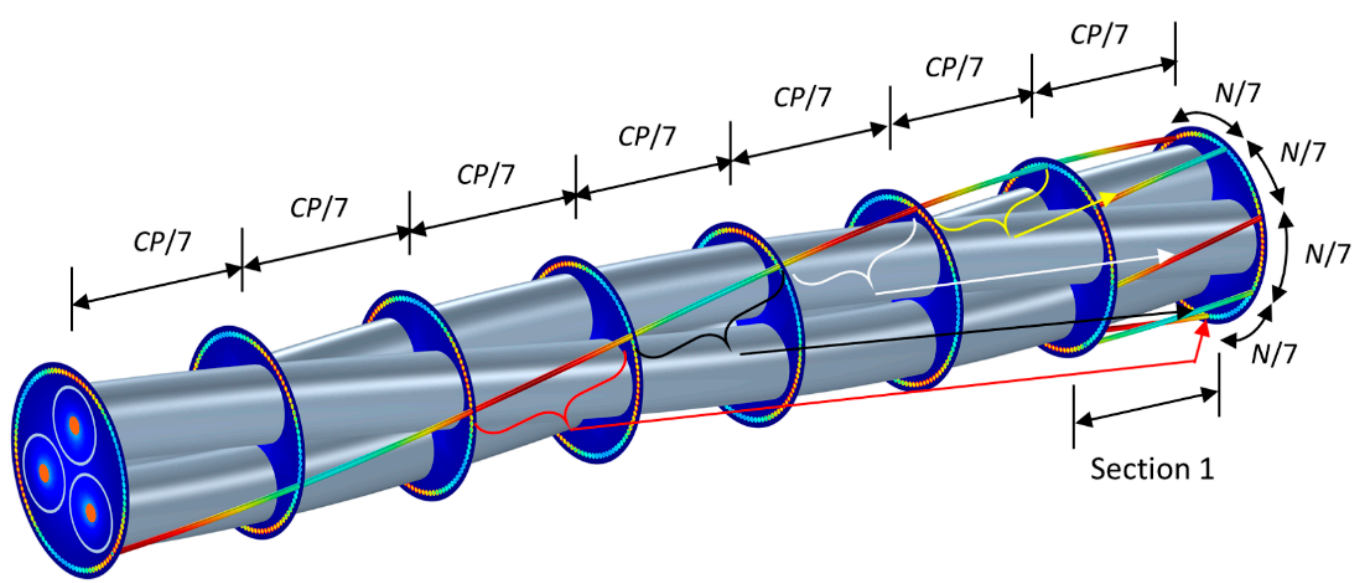

Figure 2. $C P$-length three-core cable $\left(115 \mathrm{kV}, 240 \mathrm{~mm}^{2}\right.$ copper conductor): loss color plot in one single wire and its correspondence with seven $C P / 7$-section wires in Section 1.

All this can be done for all $n$ possible values, as shown in Figure 3, where the armor wires required to reproduce the full loss pattern of the single CP-length armor wire of Figure 2 are depicted. Moreover, the length of the resulting ultra-shortened geometry (depending on $n$ ) is also indicated. To reproduce the full behavior of the armor, the same methodology must be applied to the remaining armor wires, except for $n=N$, since the resulting shortened geometry already contains the full information. Consequently, only a $C P / n$-length section of the cable is required to have all the phenomena involved in the three-core cable.
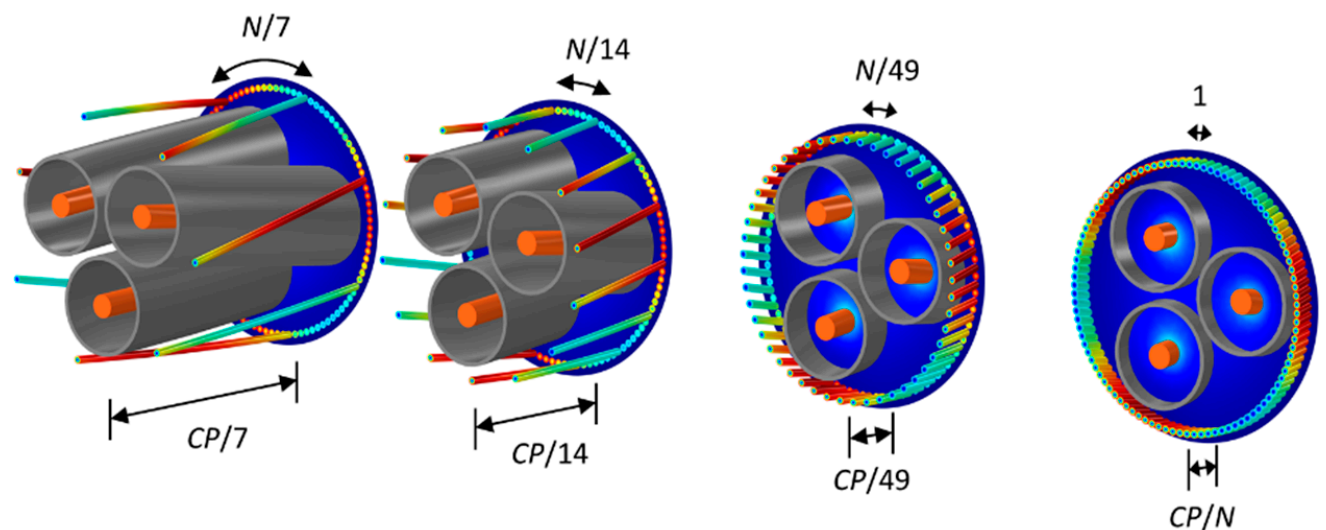

Figure 3. Armor wires required for reproducing the full pattern of one single armor wire for all $n$ values and the length of the resulting geometry.

It is to be noticed that this pattern is also observed in vector magnitudes, such as the magnetic flux density inside the armor wires, so this approach is fully valid and contains all the physical interactions involved in the power cable.

Taking all this into account, boundary conditions for applying rotated periodicity must be adequately set for a perfect matching between the source and destination boundaries (defined in Figure 4). This is achieved by assigning coordinate systems $\left(\vec{e}_{x}^{s}, \vec{e}_{y}^{s}, \vec{e}_{z}^{s}\right)$ and 
$\left(\vec{e}_{x}^{d}, \vec{e}_{y}^{d}, \vec{e}_{z}^{d}\right)$ to both boundaries, being the destination boundary system obtained by rotating the source coordinate system a certain angle $\beta$ that is a function of $n$, as

$$
\begin{gathered}
\vec{e}_{x}^{d}=\cos \beta \cdot \vec{e}_{x}^{s}+\sin \beta \cdot \vec{e}_{y}^{s}, \vec{e}_{y}^{d}=-\sin \beta \cdot \vec{e}_{x}^{s}+\cos \beta \cdot \vec{e}_{y}^{s}, \quad \vec{e}_{z}^{d}=\vec{e}_{z}^{s} \\
\beta= \pm 2 \pi \frac{C P}{n L_{c}}
\end{gathered}
$$

where the minus sign is for phases twisted counterclockwise and the positive sign for phases twisted clockwise.

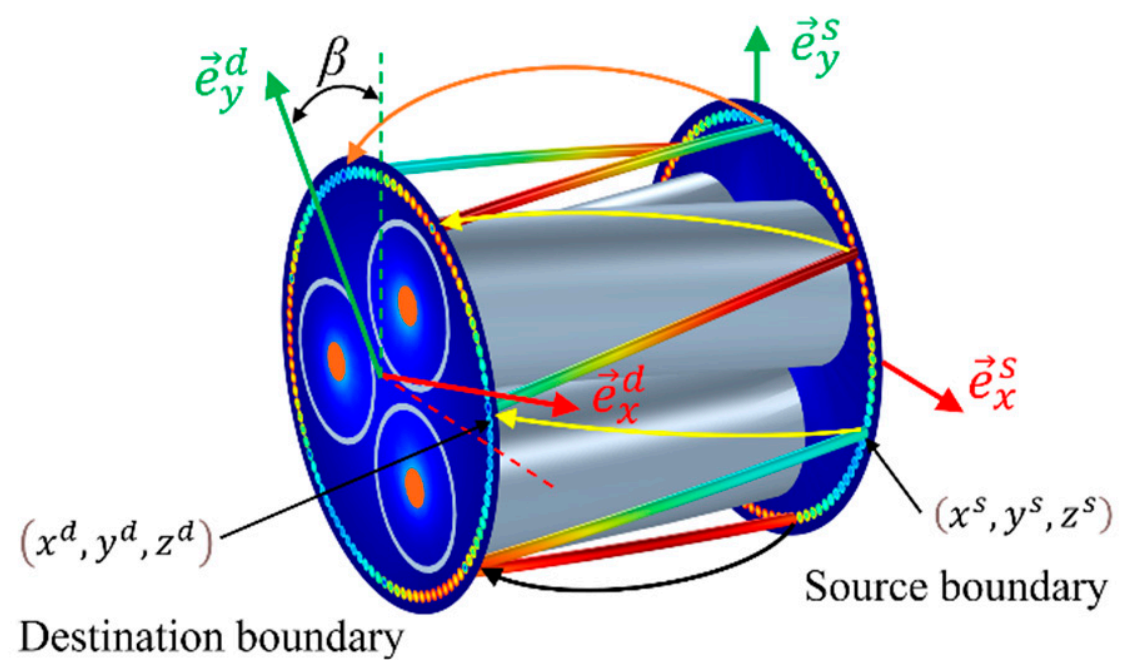

Figure 4. Rotated boundary conditions in a TCAC for a model length of $C P / 7$.

This way, the electromagnetic problem is analyzed by solving

$$
\nabla \times\left(\frac{1}{\mu} \nabla \times \vec{A}\right)+j \omega \sigma \vec{A}=\vec{J}_{e}
$$

being $\vec{A}$ the magnetic vector potential, $\sigma$ the conductivity, $\mu$ the magnetic permeability and $\vec{J}_{e}$ the external current density. Rotated periodicity is applied by means of

$$
\vec{A}\left(x^{d}, y^{d}, z^{d}\right)=\vec{A}\left(x^{s}, y^{s}, z^{s}\right)
$$

where $\left(x^{d}, y^{d}, z^{d}\right)$ and $\left(x^{s}, y^{s}, z^{s}\right)$ are the coordinates of matching points in the destination and source coordinate systems, respectively (Figure 4).

An example of the improvement achieved by the proposed approach is presented next for the same cable of $115 \mathrm{kV}$. Its main dimensions and material properties are shown in Table 1 , where $d_{c}$ is the conductor diameter, $d_{a}$ is the armor wire diameter and $D_{a}$ is the mean diameter of the armor.

Table 1. Main parameters of a $115 \mathrm{kV}, 240 \mathrm{~mm}^{2} \mathrm{TCAC}$.

\begin{tabular}{cccccccc}
\hline$d_{c}(\mathbf{m m})$ & $d(\mathbf{m m})$ & $s(\mathbf{m m})$ & $D_{a}(\mathbf{m m})$ & $d_{a}(\mathbf{m m})$ & $N$ & $L_{c}(\mathbf{m})$ & $L_{a}(\mathbf{m})$ \\
\hline 18.4 & 75.4 & 85.7 & 196 & 6 & 98 & 3.5 & 3.1 \\
\hline \multicolumn{3}{c}{$\rho(\Omega \cdot \mathbf{m})\left(\theta=20^{\circ} \mathrm{C}\right)$} & \multicolumn{3}{c}{$\mu_{r}$} \\
\hline \multicolumn{2}{c}{ Conductor } & \multicolumn{2}{c}{ Sheath } & \multicolumn{2}{c}{ Armor } \\
$1.72 \cdot 10^{-8}$ & \multicolumn{2}{c}{$2.14 \cdot 10^{-7}$} & \multicolumn{2}{c}{$1.38 \cdot 10^{-7}$} & & \multicolumn{2}{c}{$100-j 50$} \\
\hline
\end{tabular}


For this cable, Table 2 shows the most relevant simulation data and the main results obtained for different values of $n$, where $R$ and $X$ represent the cable positive-sequence resistance and the inductive reactance, $I_{S}$ is the sheath current, and $P_{c}, P_{S}$ and $P_{a}$ are the conductor, sheath and armor losses, respectively. Additionally, relevant data regarding DoF, mesh size and quality, and the RAM memory employed for the simulations are included. Simulations have been performed in a laptop with an i7 processor and 64 GB of RAM memory. It must be remarked that for $n=1$ a coarser mesh was necessary for keeping the RAM memory requirements below the available 64 GB. Instead, finer meshes were employed for the rest of the cases, increasing the mesh density with $n$. This improves the average and the minimum element quality (Table 2, where 1 is the highest quality), and hence the overall quality of the mesh.

Table 2. Results for different values of $n$ in a $115 \mathrm{kV}, 240 \mathrm{~mm}^{2} \operatorname{TCAC}\left(\theta=20^{\circ} \mathrm{C}\right)$.

\begin{tabular}{|c|c|c|c|c|c|}
\hline$n$ & 1 & 7 & 14 & 49 & 98 \\
\hline $\begin{array}{l}\text { Simulation } \\
\text { time (s) }\end{array}$ & 620 & 180 & 120 & 55 & 30 \\
\hline DoF & $6,752,506$ & $2,163,527$ & $2,023,948$ & $1,600,579$ & 787,832 \\
\hline $\begin{array}{c}\text { Mesh } \\
\text { elements }\end{array}$ & 887,260 & 273,986 & 256,663 & 204,419 & 94,442 \\
\hline $\begin{array}{l}\text { Average } \\
\text { element } \\
\text { quality }\end{array}$ & 0.5097 & 0.5567 & 0.5957 & 0.6363 & 0.6418 \\
\hline $\begin{array}{l}\text { Minimum } \\
\text { element } \\
\text { quality }\end{array}$ & $7 \cdot 10^{-4}$ & 0.0125 & 0.055 & 0.06 & 0.068 \\
\hline RAM (GB) & 60 & 22 & 17 & 9 & 4 \\
\hline$R(\Omega / \mathrm{km})$ & 0.0860 & 0.0859 & 0.0858 & 0.0858 & 0.0858 \\
\hline$X(\Omega / \mathrm{km})$ & 0.1585 & 0.1590 & 0.1591 & 0.1592 & 0.1592 \\
\hline$I_{c}(\mathrm{~A})$ & & & 500 & & \\
\hline$I_{S}(\mathrm{~A})$ & 106.25 & 107 & 107.25 & 107.35 & 107.40 \\
\hline$P_{c}(\mathrm{~W} / \mathrm{m})$ & 50.265 & 50.213 & 50.204 & 50.198 & 50.094 \\
\hline$P_{S}(\mathrm{~W} / \mathrm{m})$ & 12.208 & 12.127 & 12.107 & 12.088 & 12.002 \\
\hline$P_{a}(\mathrm{~W} / \mathrm{m})$ & 2.0857 & 2.0735 & 2.074 & 2.0764 & 2.0773 \\
\hline$\lambda_{1}(\mathrm{FEM})$ & 0.2428 & 0.2415 & 0.2412 & 0.2408 & 0.2396 \\
\hline$\lambda_{1}[5]$ & & & 0.246 & & \\
\hline$\lambda_{2}(\mathrm{FEM})$ & 0.0415 & 0.0413 & 0.0413 & 0.0414 & 0.0415 \\
\hline$\lambda_{2}[5]$ & & & 0.221 & & \\
\hline
\end{tabular}

As can be seen, differences in the results derived for each value of $n$ are virtually negligible, and the reduction achieved in computation time for the case of $n=N$ is to be remarked, with just $30 \mathrm{~s}$ for solving such a complex model and with no loss in accuracy. Consequently, a model length equal to $C P / N$ is the best choice. Further, it is of interest to highlight how there is a good agreement for this cable between the $\lambda_{1}$ values from FEM and [5]. However, important differences are observed in $\lambda_{2}$, as previously reported in $[3,4]$.

\subsection{Loss Allocation}

For a better understanding of all the phenomena involved in loss generation, as well as analyzing its dependence with technical and material parameters, some additional improvements have been implemented in the 3D-FEM model.

\subsubsection{Comparing Results}

The FEM software employed in this study allows for making simple numerical operations with the solutions, of any spatial magnitude or field, obtained for the same geometry but considering two different sets of boundary conditions (C1 and C2). This feature is used here to define a ratio $\gamma$ for evaluating, on each point of the cable geometry $(x, y, z)$, 
the variation of a certain magnitude $\xi$ when boundary conditions are changed from $C 1$ to C2, as

$$
\gamma=\frac{\xi_{\mathrm{C} 2}(x, y, z)}{\xi_{\mathrm{C} 1}(x, y, z)}
$$

When plotted over the cable geometry this ratio allows for visualizing the regions of the cable where $\xi$ increases $(\gamma>1)$ or decreases $(\gamma<1)$ in relation to the reference situation (C1). An example is shown in Figure 5 for a $275 \mathrm{kV}, 2000 \mathrm{~mm}^{2}$ three-core unarmored cable (Table 3), where it is represented the variation that takes place in the conductor losses $\left(\xi=P_{c}\right)$ when switching from sheaths in SP $(C 1)$ to SB $(C 2)$.

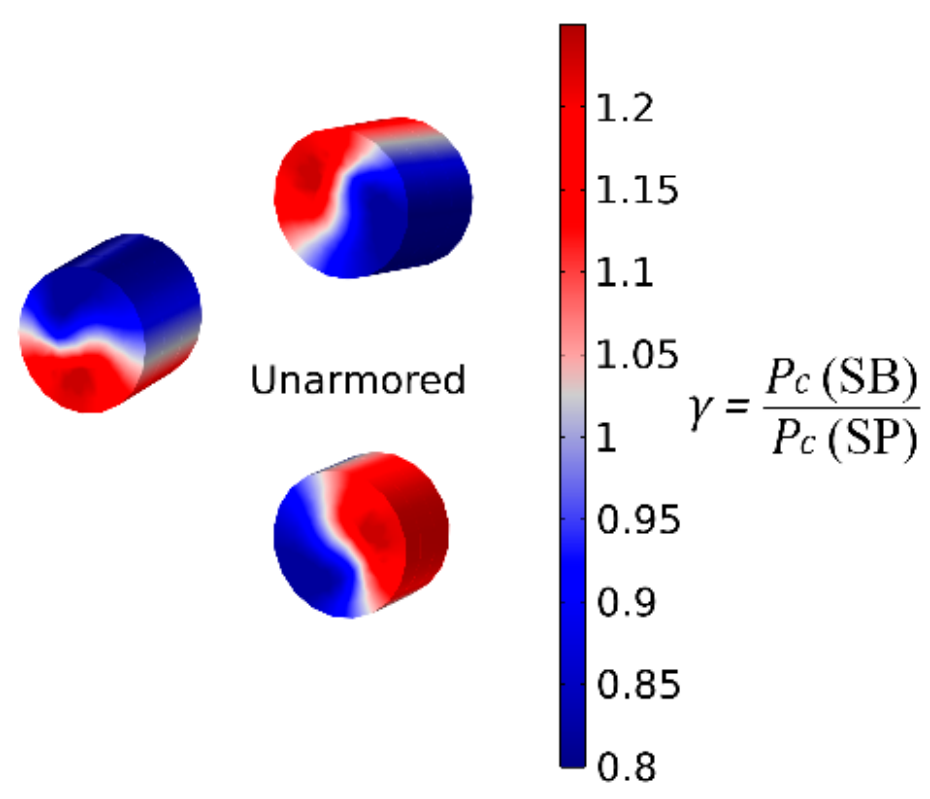

Figure 5. Color plot of the ratio of conductor losses from SP bonding scheme to SB $\left(2000 \mathrm{~mm}^{2}, 275 \mathrm{kV}\right.$ unarmored cable, phase current of $1100 \mathrm{~A})$.

From Figure 5 it can be concluded that the current density in the conductors is affected by the bonding scheme, leading to an increase in losses of more than $20 \%$ in half of the conductor when changing to $\mathrm{SB}$, while it decreases in less than $20 \%$ in the remaining regions. The extension of each region results in a global increase or decrease in $\xi$ ( $P_{c}$ in this example). In this case, since the current through the conductors is the same in $C 1$ and $C 2$, the average value of $\gamma$ can be taken as the factor in which the conductor resistance is affected by changes in the bonding configuration. In this way it is easier to evaluate qualitatively and quantitatively how variations in the boundary conditions influence the conductor resistance.

\subsubsection{Separation of Circulating and Eddy Losses}

As mentioned before, there is a controversial point regarding loss allocation in the sheaths, since [5] assumes that eddy losses may be neglected when sheaths are in SB, although some studies suggest the opposite for large cables $[6,7,33,34]$. Therefore, to clarify this point it is required to find a way to evaluate these terms separately, especially in TCACs. In this case, the induced currents mainly follow a helical path due to the conductor twisting (Figure 6a for a $275 \mathrm{kV}, 2000 \mathrm{~mm}^{2}$ three-core unarmored cable). Therefore, it is expected that eddy losses differ from those derived from Equation (7), which is intended for three parallel and straight single-core cables. Moreover, the presence of a magnetic armor is not considered in this expression. 
Table 3. Main parameters for test cables.

\begin{tabular}{ccccccc}
\hline Parameter & Cable 1 & Cable 2 & Cable 3 & Cable 4 & Cable 5 & Cable 6 \\
\hline Voltage $(\mathrm{kV})$ & 115 & 132 & 150 & 220 & 220 & 275 \\
Section $\left(\mathrm{mm}^{2}\right)$ & 400 & 800 & 630 & 1200 & 1600 & 2000 \\
Conductor & $\mathrm{Cu}$ & $\mathrm{Cu}$ & $\mathrm{Cu}$ & $\mathrm{Al}$ & $\mathrm{Cu}$ & $\mathrm{Cu}$ \\
Current $(\mathrm{A})$ & 530 & 730 & 650 & 900 & 900 & 1100 \\
Conductor diam. $(\mathrm{mm})$ & 23.5 & 35 & 30.25 & 42.9 & 46.3 & 54.5 \\
Sheath ext. diam. $(\mathrm{mm})$ & 78.7 & 87.6 & 80.6 & 99.5 & 104 & 121.5 \\
Sheath thickness $(\mathrm{mm})$ & 3.3 & 3.7 & 2.8 & 2.25 & 2.25 & 3 \\
Armor mean diam. $(\mathrm{mm})$ & 49.5 & 212 & 195 & 231.2 & 241.5 & 295.6 \\
Armor wire diam. $(\mathrm{mm})$ & 6 & 5.6 & 6 & 5 & 5.6 & 5.6 \\
No. armor wires & 98 & 114 & 95 & 139 & 129 & 157 \\
Core diam. $(\mathrm{mm})$ & 85.7 & 92.4 & 85.6 & 104.4 & 108.9 & 126 \\
Core lay length $(\mathrm{m})$ & 2.5 & 2.8 & 2.6 & 3.6 & 3.6 & 3.8 \\
Armor lay length $(\mathrm{m})$ & 3.1 & 3.5 & 1.8 & 4 & 4 & 4.8 \\
\hline
\end{tabular}

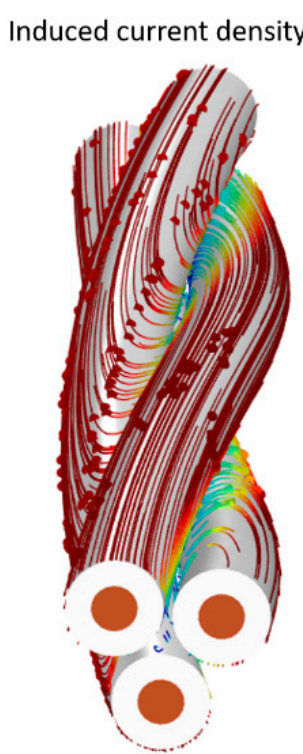

(a)

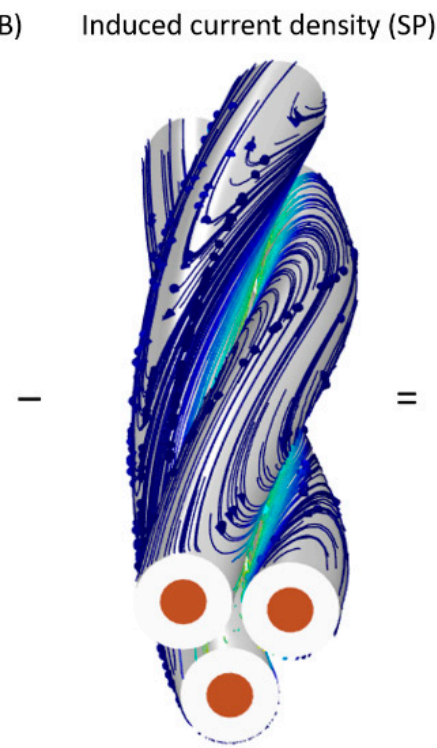

(b)

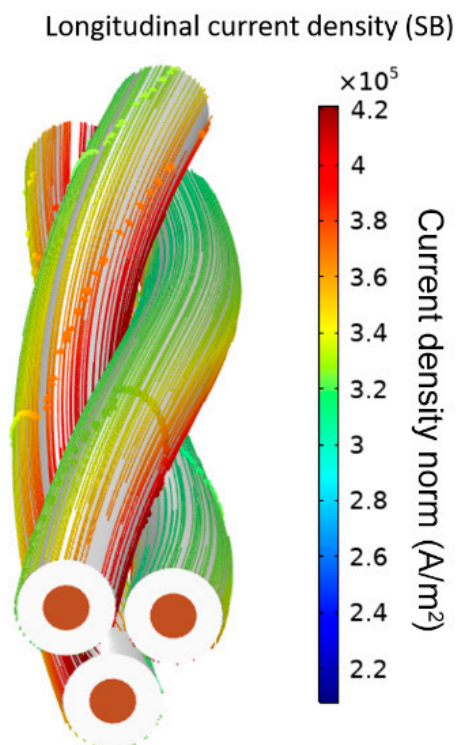

(c)

Figure 6. Procedure employed in the FEM software for obtaining the circulating current density (c) by subtracting the SP eddy-current (b) from the total SB induced current (a) $\left(2000 \mathrm{~mm}^{2}, 275 \mathrm{kV}\right.$ unarmored cable, phase current $1100 \mathrm{~A}$ ).

Having this in mind, it is here proposed a way to evaluate sheath circulating and eddy losses separately by means of 3D-FEM simulations. To this aim, it is assumed that the external flux density causing eddy-current losses is not substantially affected by the bonding scheme, i.e., the same eddy-current distribution is expected in the sheaths. Thus, the 3D-FEM model of the cable is first solved for sheaths in SP and SB. Once done, the FEM software allows subtracting the eddy-current density distribution obtained in the SP-cable $\left(\vec{J}_{S P}\right)$ from that derived for the SB situation $\left(\vec{J}_{S B}\right)$, as shown in Figure 6 . This results in a current density distribution $\left(\vec{J}_{\text {long }}\right)$ that absolutely follows the helical path of the sheaths (Figure $6 \mathrm{c}$ ). Furthermore, the resulting net current is also equal to that measured in the sheaths for the SB-cable, thus verifying our assumption. This current density represents the cause of the circulating losses $\left(P_{s}^{\prime}\right)$ which can be evaluated integrating through the three sheath volumes by

$$
P_{s}^{\prime}=\frac{\rho_{s}}{2} \int \vec{J}_{\text {long }}^{2} d V
$$

where $\rho_{s}$ is the sheath resistivity. 
On the other hand, since the net current flowing through the sheaths can be computed $\left(I_{S}\right)$, it is also possible to obtain the AC resistance of the sheaths from $P_{s}^{\prime}$ as

$$
R_{s}=\frac{P_{S}^{\prime}}{3 I_{S}^{2}}
$$

Finally, since the global sheath losses in SB can be also derived from $\vec{J}_{S B}$ by Equation (17), it is now possible to obtain eddy losses $\left(P_{s}^{\prime \prime}\right)$ as

$$
P_{s}^{\prime \prime}=P_{s}-P_{s}^{\prime}
$$

An in-depth analysis of the results derived from this method will be discussed in the following sections.

\section{Analysis of Conductor and Sheath Losses}

Inaccurate results are derived from [5] since $R_{s}, X_{s}$ and $R_{c}$ are obtained by ignoring important aspects related to TCACs, such as the core and armor twisting, the bonding scheme, as well as the magnetic properties of the armor wires. The influence of these aspects on $P_{c}$ and $P_{S}$ is analyzed in this section, being these two losses individually evaluated by means of the improved 3D-FEM model (Figure 7). Moreover, the proposals presented in previous works $[6,10,29,31]$ are also evaluated.

Conductor losses $\left\{\begin{array}{l}\begin{array}{l}\text { Effect of core twisting } \\ \text { (neither armor nor sheaths) } \\ \text { Effect of sheath bonding } \\ \text { (no armor) } \\ \text { Effect of armor and sheaths } \\ \text { (sheaths only in SB) }\end{array}\end{array}\right.$

(a)
Sheath losses $\left\{\begin{array}{l}\text { Effect of twisting and armor } \\ \text { Sheath current magnitude }\end{array}\right.$

(b)

Figure 7. Analysis developed in Section 4 for $(\mathbf{a})$ conductor losses $\left(P_{c}\right)$ and $(\mathbf{b})$ sheath losses $\left(P_{s}\right)$.

As a result, different approaches for improving both the conductor and sheath losses are proposed. To this aim, 6 test cables are employed (Table 3), although most of the analyses throughout the paper are illustrated using the largest cable (Cable 6) for clarity. The electrical resistivity of the conductive parts is taken from [5] for an ambient temperature of $20^{\circ} \mathrm{C}$. A complex relative permeability in the form of $\mu_{r}=\mu^{\prime}-j \mu^{\prime \prime}$ (ranging from $1-j 0$ (no armor/stainless steel armor) to $600-j 350$ ) is employed for the armor wires in order to consider hysteresis losses.

\subsection{Conductor Losses}

The effects of core twisting, sheath bonding and the armor on the conductor losses (not considered in [5]) are analyzed next.

\subsubsection{Core Twisting}

The influence of the core twisting is shown in Figure 8 for Cable 6 when neither armor nor sheaths are present, where the evolution of $P_{\mathcal{c}}$ (Figure $8 \mathrm{a}$ ) and $R_{\mathcal{c}}$ (Figure $8 \mathrm{~b}$ ) with $L_{\mathcal{c}}$ are represented (Cable $6 L_{c 6}$ is marked with a vertical line). It is easily seen how the values of $R_{c}$ and $P_{c}$, derived from FEM simulations, increase with the twisting of the conductors. This leads to important differences with those derived by the IEC standard (labelled as " $P_{c}$ IEC"), especially when phases are more twisted (shorter lay length), where it is more evident that [5] underestimates their values. 


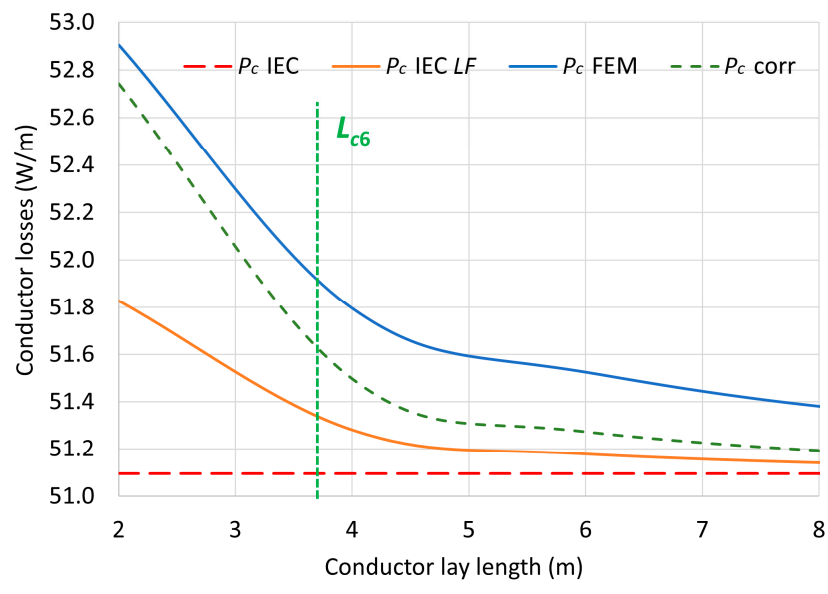

(a)

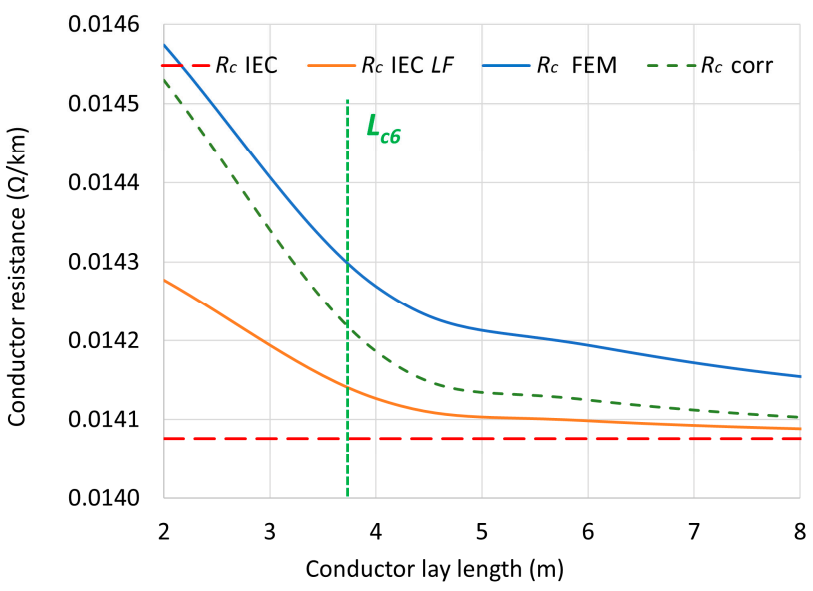

(b)

Figure 8. Conductor (a) losses and (b) resistance for Cable 6 with neither armor nor sheaths $\left(20^{\circ} \mathrm{C}\right)$.

As recommended in [29], the additional length of the conductor due to the twisting of the phases should be considered in the analytical computation of the DC resistance. This is done by multiplying Equation (3) by LF (previously defined in Equation (9)). This correction in [5] leads to better results in Figure 8 (denoted by " $P_{c}$ IEC $L F$ " and " $R_{c}$ IEC $L F^{\prime \prime}$, respectively), since lower differences with FEM results are observed.

Nonetheless, some differences are still observed. This might be explained as an increase in the proximity effect due to the conductor twisting, which leads to a slightly higher $\mathrm{AC}$ resistance. In this sense, since $y_{p}$ is expressed as a function of $d_{c} / s$, a first hypothesis is that the concepts of conductor-conductor distance and conductor diameter must be re-defined in case of twisting. To better visualize this, Figure 9 shows the resulting cross section obtained by a cut plane orthogonal to one of the phases (indicated by a green arrow). As can be seen, while the cross section of one conductor remains circular in that plane, the other two are in fact ellipses, where the major axis is higher that the conductor diameter in $L F^{3}$, and the minor axis remains equal to $d_{c}$. Moreover, in that plane the distance between the axes of circular and elliptical conductors is $s \cdot \cos \alpha / 2$, being

$$
\alpha=\operatorname{atan}\left(\sqrt{L F^{2}-1}\right) .
$$

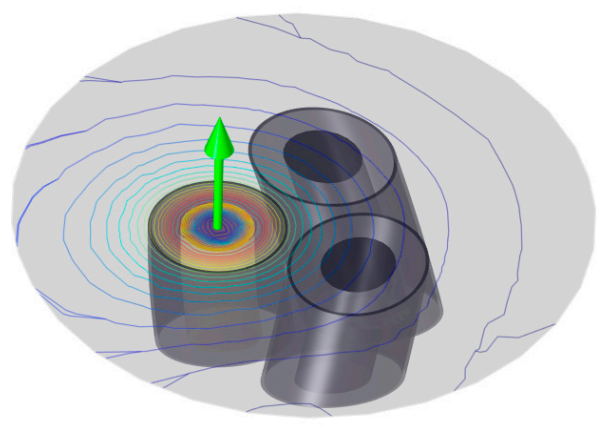

(a)

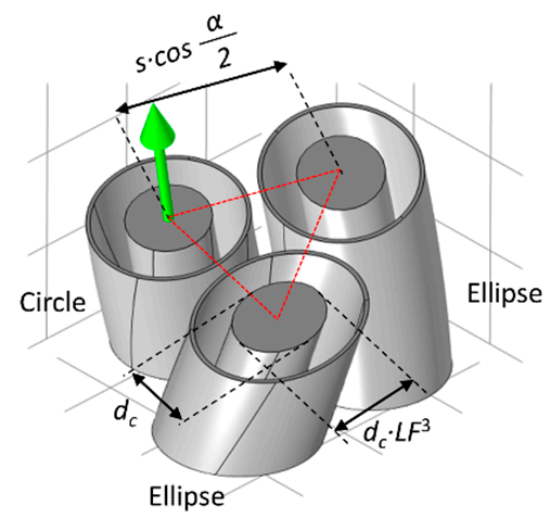

(b)

Figure 9. (a) Resulting cross section obtained by a cut plane orthogonal to the green vector in the direction of one of the phases and $(\mathbf{b})$ distances and dimensions inside the cut plane. 
All this influences the magnetic flux concatenated by the elliptical conductors (Figure 9a), reinforcing the proximity effect. Consequently, we propose to multiply every term $d_{c} / s$ in the expression of $y_{p}$ (given in [5]) by a coefficient $f_{t}$ as follows

$$
\begin{gathered}
y_{p}^{\prime}=\frac{3\left(\frac{d_{c}}{s} f_{t}\right)^{2} G\left(x_{p}\right)}{2-\frac{5}{12}\left(\frac{d_{c}}{s} f_{t}\right)^{2} H\left(x_{p}\right)} \\
f_{t}=\frac{L F^{3}}{\cos \frac{\alpha}{2}},
\end{gathered}
$$

where $x_{p}, G\left(x_{p}\right)$ and $H\left(x_{p}\right)$ are defined in [5].

After applying this approach, differences with FEM results decrease even more, as observed in Figure 8 (denoted by " $P_{c}$ corr" and " $R_{c}$ corr", respectively).

\subsubsection{Effect of Sheath Bonding}

The presence of sheaths in a three-core cable (either SP or SB) also influences the conductor losses. This is shown in Figure 10a, where 3D-FEM results for $P_{c}$ are represented for Cable 6 (Table 3, but unarmored) as a function of $L_{c}$. Additionally, the case where the three-core cable has no sheaths is included (denoted by " $P_{c}$ ns").

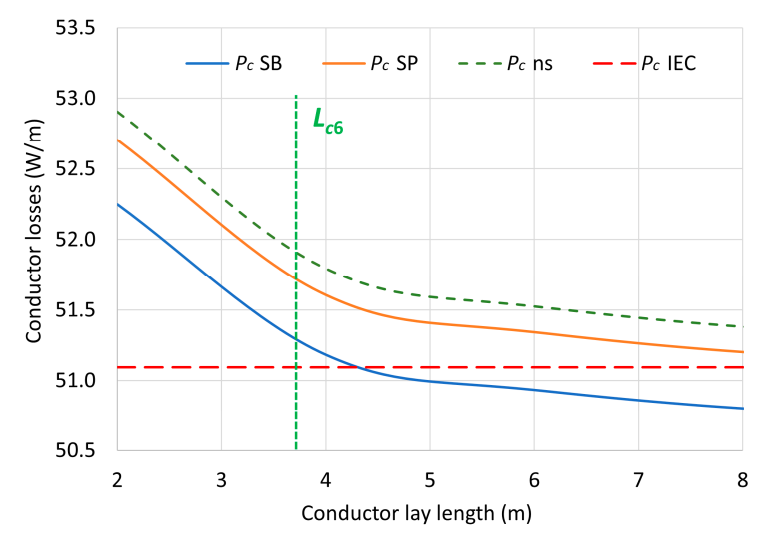

(a)

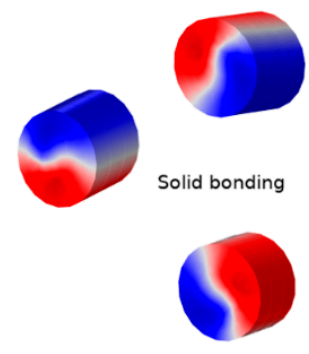

(b)

Figure 10. (a) Evolution of the conductor losses as a function of the lay length for SP, SB and with no sheaths, and (b) variations in the conductor current density distribution due to the presence of the sheaths in Cable 6 (unarmored, phase current $1100 \mathrm{~A}$ ).

As can be observed, the value of $P_{c}$, and hence its resistance, is higher when no sheaths are installed, and it reduces in presence of sheaths, especially when connected in SB, being more noticeable when phases are more twisted. A possible answer to this behavior may be found in Figure 10b, where the ratio $\gamma$ is depicted for the current density $J$ from absence $\left(J_{c}^{n s}\right)$ to presence $\left(J_{c}^{s}\right)$ of shields. Thus, it is observed how, in both SB and SP, in half of the conductor cross section the current density increases over the situation with no sheaths, while it decreases in the other half. However, these variations are more intense in the SB case, reaching up to $\pm 15 \%$. In this sense, an average evaluation of $\gamma$ in the conductor volume results in a factor of 0.99 for the SP case and 0.98 for the SB situation, in accordance with the results observed in Figure 10a. Anyway, these values are quite low, so its influence can be neglected (this effect will not be negligible with armor, as analyzed next).

\subsubsection{Combined Effect of Magnetic Armor and Sheath Bonding}

As previously indicated, to analyze the influence of the magnetic armor on $R_{c}$ and $P_{c}$, only sheaths in SB are considered, since it is the preferred bonding configuration in 
offshore applications [30]. This way, the results derived by FEM simulations for Cable 6 are represented in Figure 11, where different values of $\mu_{r}, L_{a}$ and $L_{c}$ are considered (the actual values $L_{c 6}$ and $L_{a 6}$ of Cable 6 are also shown). Figure 11 also includes the results obtained in the unarmored case, together with those derived by [5] and the approaches proposed in previous subsections (Equations (9) and (21)) (labelled as " $P_{c}$ corr" in Figure 11).

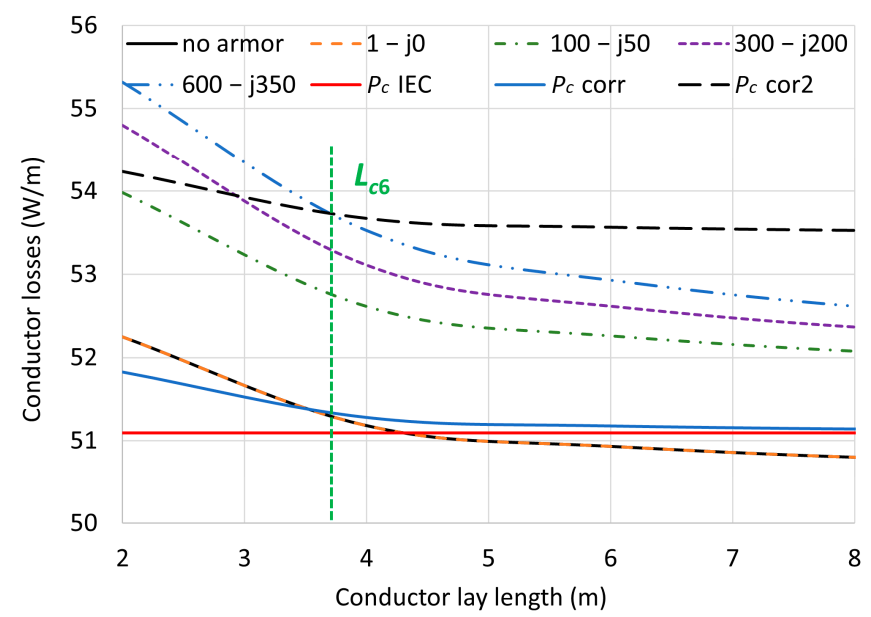

(a)

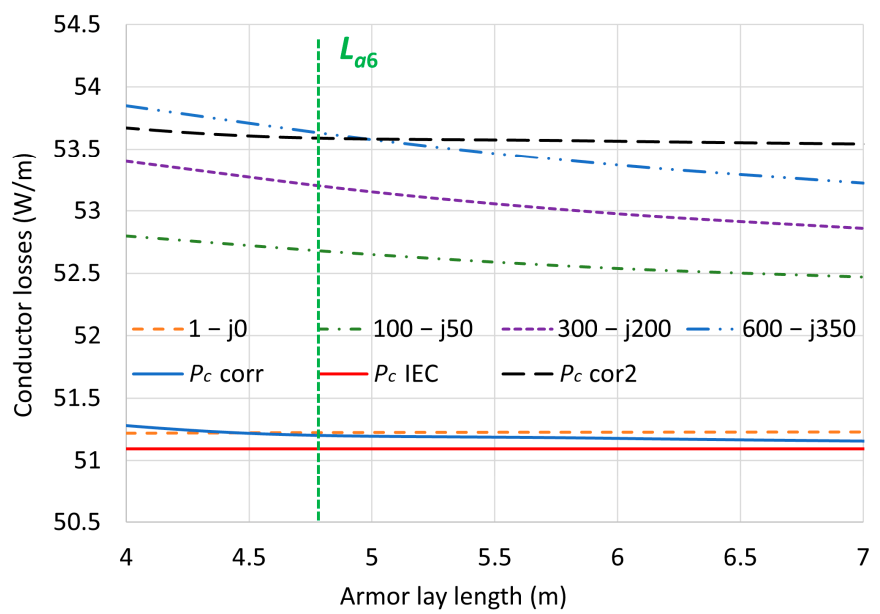

(b)

Figure 11. Variation of conductor losses with the armor permeability and (a) the conductor twisting or (b) the armor twisting in Cable $6(\mathrm{SB}) . L_{c 6}\left(L_{a 6}\right)$ : actual conductor (armor) lay length.

A first conclusion arises from the results regarding the stainless-steel armor $\left(\mu_{r}=1-j 0\right)$, since it has no effects on the conductor losses as the results overlap with those obtained for an unarmored cable. In both cases the results are quite close to those derived by [5], especially when the corrections previously suggested are applied (" $P_{c}$ corr"). However, in presence of a magnetic armor a different behavior is observed, since $P_{c}$ increases with $\mu_{r}$, especially when conductors are more twisted (Figure 11a), leading to important differences with regard to the values provided by [5]. Furthermore, the lay length of the armor also influences the conductor losses, but to a lesser extent (Figure 11b).

The reasons for these results come again from the use of $\gamma$. In this case, Figure 12a,b illustrates, respectively, the changes in the conductor losses and the current density distribution when switching from absence $\left(P_{c}^{n a}, J_{c}^{n a}\right)$ to presence $\left(P_{c}^{a}, J_{c}^{a}\right)$ of armor. The results show how both losses and current density increase in areas close to the center of the cable, whereas it strongly decreases in the most external regions of the conductors. This can be understood as an increase in the proximity effect inside the conductors since the current density moves towards the inner region of the cable. This conclusion is in line with recent recommendations [30] regarding the application of a factor of 1.5 to both $y_{s}$ and $y_{p}$ for including the effect of the armor on $R_{c}$. However, from this analysis it seems reasonable to apply the correction factor only to $y_{p}$, resulting in the curve denoted by " $P_{c}$ cor2" in Figure 11. By doing this, the differences relative to FEM results decrease, although conductor losses are generally overestimated. 

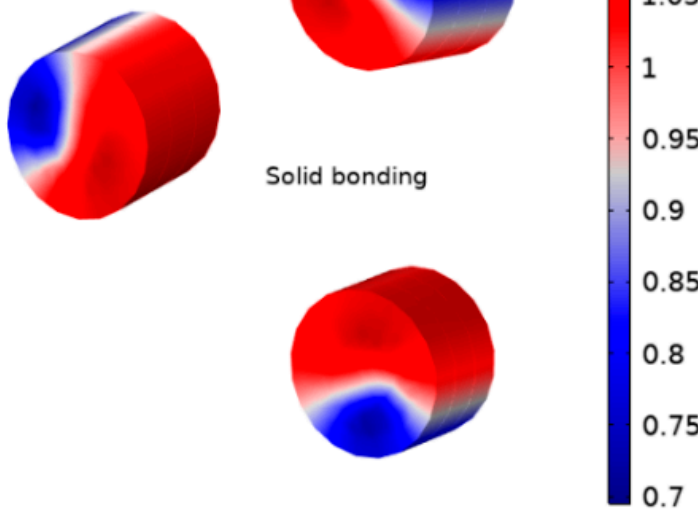

(a)
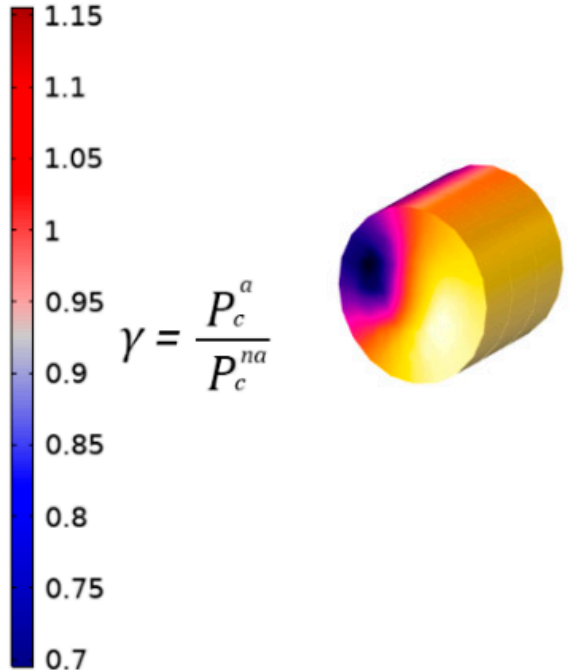
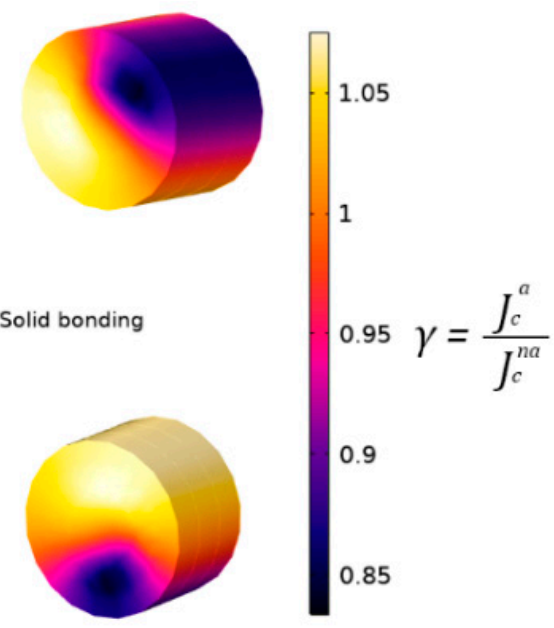

0.9

(b)

Figure 12. Variations in the (a) losses and (b) current density distribution in conductors of Cable 6 when a magnetic armor of $\mu_{r}=300-j 200$ is installed (SB).

Hence, we propose the following corrections in Equations (2) and (3) [29]:

$$
\begin{gathered}
R_{c}(\theta)=R_{c}^{D C}(\theta)\left(1+y_{s}+1.5 y_{p}^{\prime}\right), \\
R_{c}^{D C}(\theta)=\frac{\rho_{c}\left(\theta_{0}\right) L F}{S_{c}}\left(1+\alpha_{c}\left(\theta-\theta_{0}\right)\right),
\end{gathered}
$$

where $y_{p}^{\prime}$ was defined in Equation (18). The errors in $R_{c}$ (relative to FEM) obtained from this approach and [5] are represented in Figure 13 for the 6 cables and different values of $\mu_{r}$. It is easily observed how the new proposal reduces the relative differences, showing a maximum below 3\% for Cable 6, while the IEC standard underestimates the conductor resistance in up to $-6 \%$ in Cable 5 .

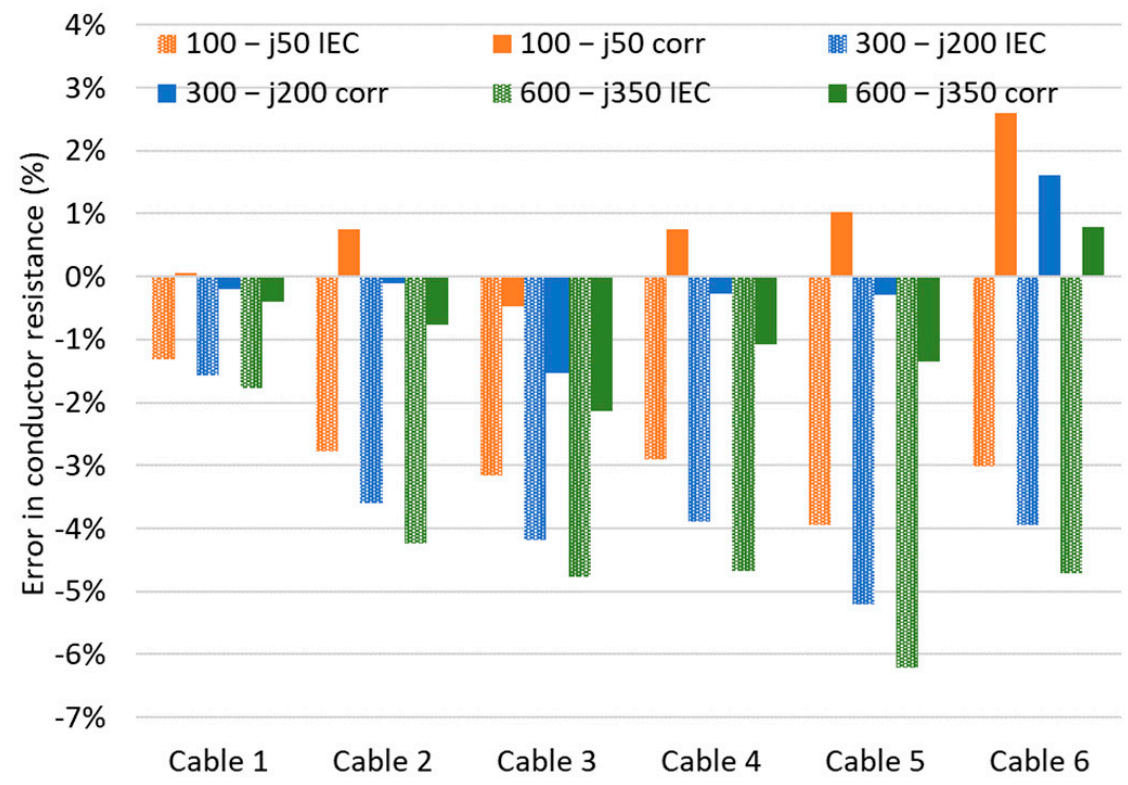

Figure 13. Error in conductor resistance, relative to FEM solutions, of the results derived from the formulation provided by [5] and the proposed approaches. 


\subsection{Sheath Losses}

Although important advances have been developed recently [6], some aspects such as the core twisting or the presence of the armor are not considered in the available formulae [5,32]. The lack of accurate expressions for this type of cable, as well as the influence of the mentioned aspects on the sheath losses are described next (only sheaths in $\mathrm{SB}$ will be considered, as commented earlier).

\subsubsection{Influence of Twisting and Armor $\mu_{r}$}

The influence of the cores and armor twisting in the total sheath losses are depicted in Figure 14a for Cable 6, where $C P$ collects in one factor different sets of values for these parameters $\left(\mathrm{CP}_{6}\right.$ of Cable 6 is marked with a vertical line). Additionally, the effect of $\mu_{r}$ is represented, as well as the values for the sheath losses derived by [5] when twisting is taken into consideration by including $L F$ in the computation of $R_{S}$ :

$$
R_{s}(\theta)=\frac{\rho_{s}\left(\theta_{0}\right) L F}{S_{s}}\left(1+\alpha_{s}\left(\theta-\theta_{0}\right)\right)
$$

where $S_{s}$ is the cross section and $\alpha_{s}$ is the temperature constant of the sheath.

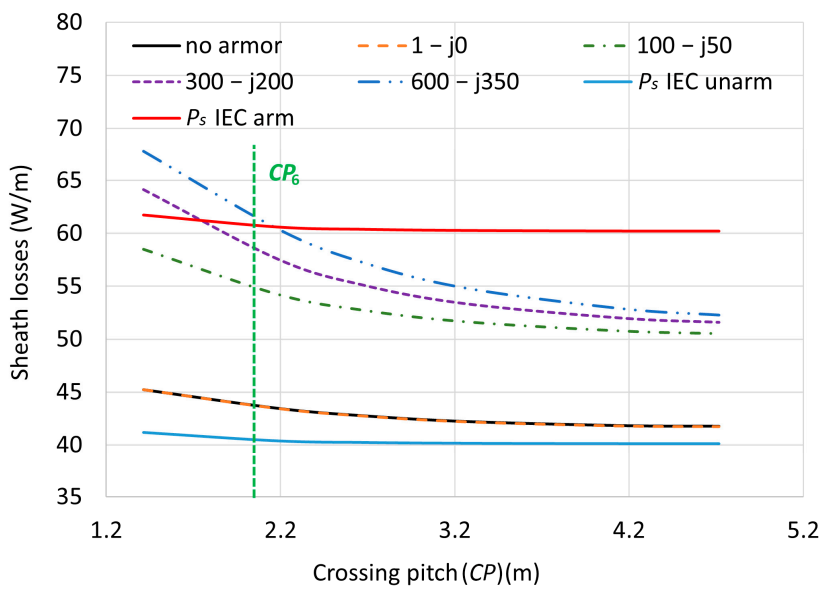

(a)

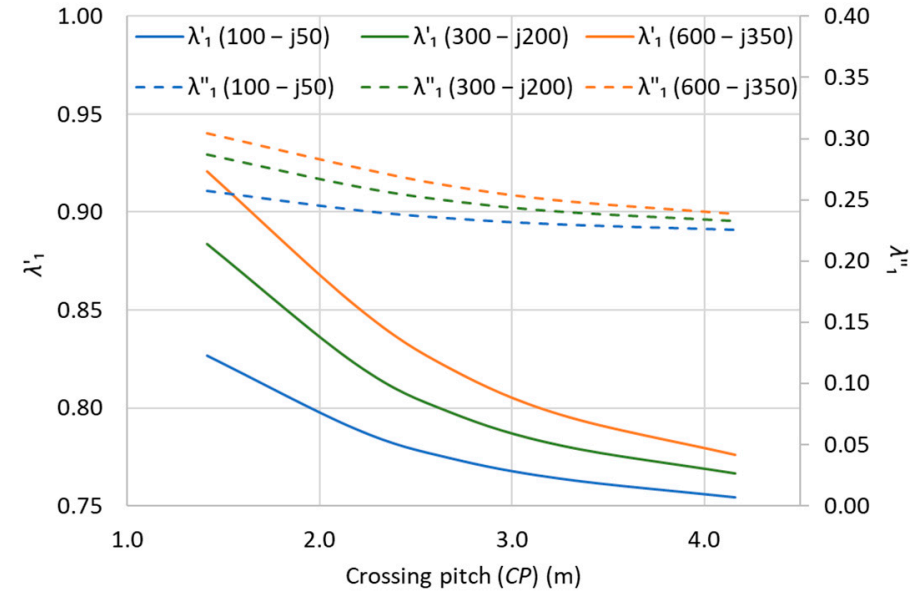

(b)

Figure 14. Evolution of (a) the total sheath losses and (b) the sheath loss factors with $C P$ and the armor permeability in Cable 6 (SB).

From Figure 14a it can be concluded that both $C P$ and $\mu_{r}$ influence the sheath losses, especially for lower values of $C P$. Although results show a good agreement between FEM and the IEC expressions for the case of the unarmored cable, it is not the case for armored ones, resulting in values generally overestimated. This is a consequence of applying an empirical factor of 1.5 in Equation (6) for computing $\lambda^{\prime}{ }_{1}$.

To analyze how circulating and eddy losses are affected by the armor, the loss factors $\lambda^{\prime}{ }_{1}$ and $\lambda^{\prime \prime}{ }_{1}$ are derived by means of the methodology proposed in Section 3.2.2. The results are represented in Figure 14b for Cable 6 as a function of $C P$ and the armor permeability. It is observed how both $\lambda^{\prime}{ }_{1}$ and $\lambda^{\prime \prime}{ }_{1}$ increase with $\mu_{r}$ and $C P^{-1}$, especially $\lambda^{\prime}{ }_{1}$ for lower values of $C P$. Similar results are derived for Cables 1 to 5 (not included for clarity).

On the other hand, the results obtained for $\lambda^{\prime}{ }_{1}$ and $\lambda^{\prime \prime}{ }_{1}$ by the analytical expressions included in [5], the new expressions proposed in [6], as well as those derived by FEM simulations, are compared in Figure 15 for the 6 cables employed in this work, where only two values for $\mu_{r}$ are considered for clarity. 


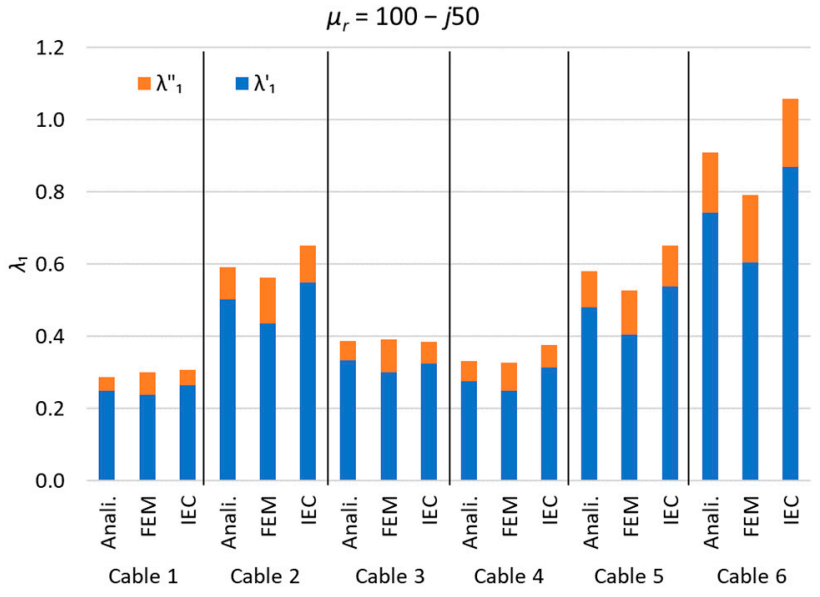

(a)

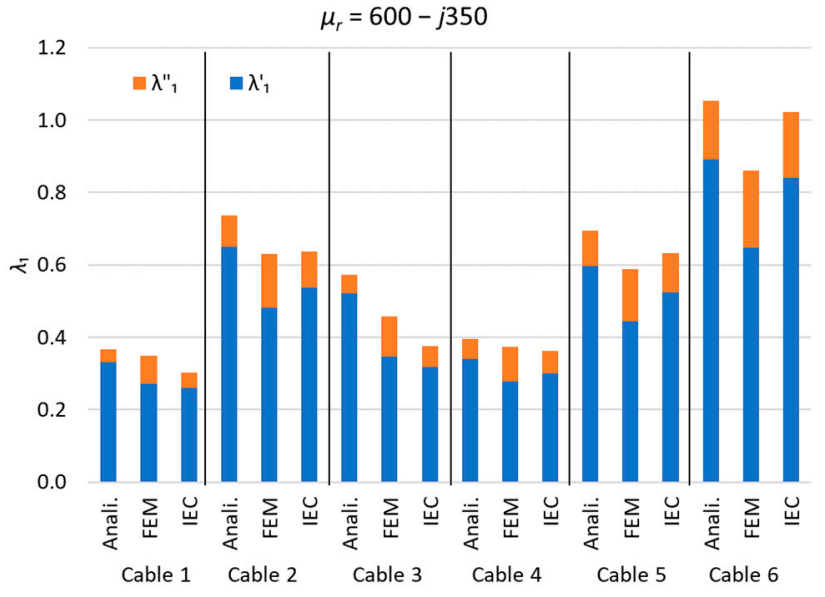

(b)

Figure 15. Circulating, eddy and total losses for the 6 cables with (a) $\mu_{r}=100-j 50$ and (b) $\mu_{r}=600-j 350$ derived by 3D-FEM simulations and the analytical expressions presented in $[5,6]$.

From the analysis of Figure 15 it is concluded that $[5,6]$ and FEM simulations provide similar results for the smaller cables (cables 1,3) and the aluminum conductor (cable 4), mainly for $\mu_{r}=100-j 50$ (Figure 15a), although [5,6] overestimate the total losses in the larger cables (cables 5, 6). It is also to be remarked that only the expressions of [6] include the effect of $\mu_{r}$ in the sheath losses, that increase with $\mu_{r}$ as expected (Figure 15b) (results obtained by [5] remain unaffected). However, it provides greater values for $\lambda_{1}^{\prime}$ than FEM, leading to an overestimation of the total losses, especially for the largest cable. Moreover, FEM simulations provide slightly greater values for $\lambda^{\prime \prime}{ }_{1}$ than $[5,6]$, although differences in $\lambda_{1}^{\prime}$ are more significant.

From these results it is also possible to analyze the relative importance of $\lambda^{\prime}{ }_{1}$ and $\lambda^{\prime \prime}{ }_{1}$ in the sheath total losses. This is shown in Figure 16, where the ratios $\lambda_{1} / \lambda_{1}^{\prime}$ (Figure 16a) and $\lambda_{1} / \lambda^{\prime \prime}{ }_{1}$ (Figure 16b), derived by FEM simulations, are represented for all 6 cables as a function of the real part of $\mu_{r}\left(\mu^{\prime}\right)$.

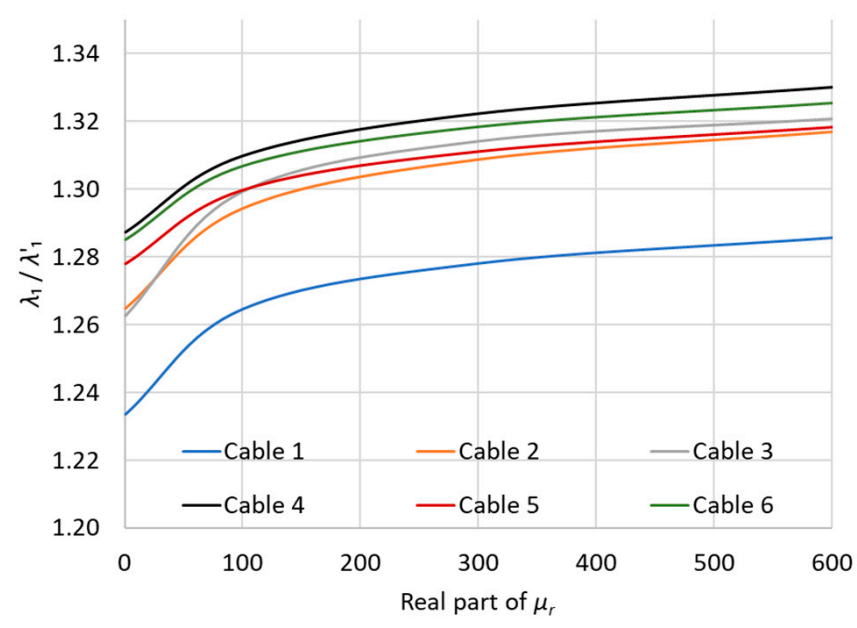

(a)

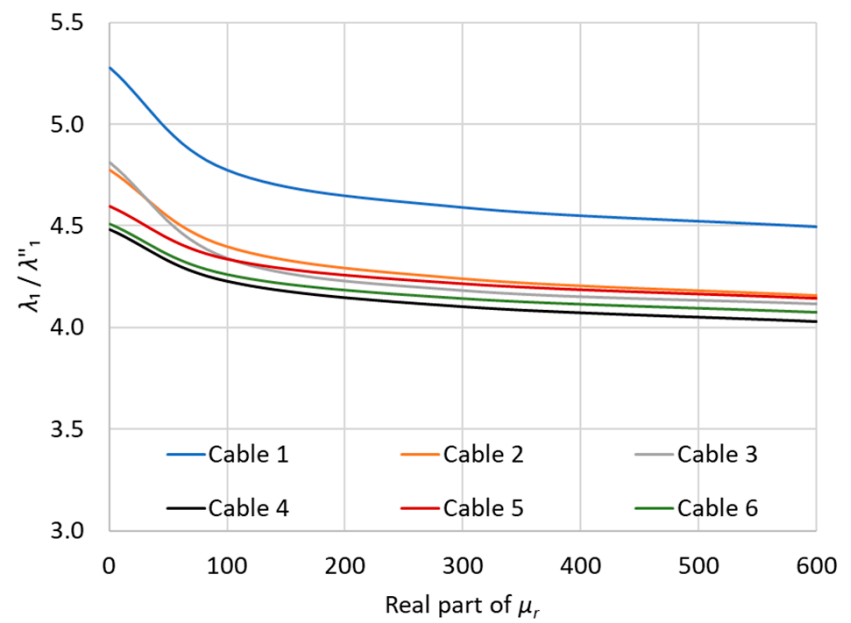

(b)

Figure 16. (a) $\lambda_{1} / \lambda^{\prime}{ }_{1}$ and (b) $\lambda_{1} / \lambda^{\prime \prime}{ }_{1}$ against real part of $\mu_{r}$, obtained from FEM simulations for the 6 cables analyzed.

It can be seen how both ratios hardly vary for $\mu^{\prime}>100$, ranging from 1.26 to 1.32 in the case of $\lambda_{1} / \lambda^{\prime}{ }_{1}$, while $\lambda_{1} / \lambda^{\prime \prime}{ }_{1}$ does it from 4 to 4.7 . Similar results are derived by [5,6], although a wider range is observed for both parameters, having $\lambda_{1}^{\prime}$ a greater relative 
weight in $\lambda_{1}$ than that observed in FEM results. In any case, these values can be taken as references for the evaluation of $\lambda_{1}$ in other armored cables.

\subsubsection{Sheath Current Evaluation}

The discrepancies observed previously in the sheath losses may be caused by differences in the sheath current calculated by each method. In this sense, previous references like $[5,38]$ only provide an expression for three single-core cables in SB, where neither the core twisting nor the presence of a magnetic armor are considered. In contrast, [6] proposes new expressions for computing $X_{s}$ that include all these aspects. In both cases the sheath current is derived as

$$
I_{S}=\frac{X_{s} I_{\mathcal{C}}}{\sqrt{R_{S}^{2}+X_{s}^{2}}} .
$$

To show how $\mu_{r}$, as well as the twisting of phase/armor (both included in the $C P$ ), influence $I_{S}$, some results derived by 3D-FEM simulations for Cable 6 are represented in Figure 17. The evolution of $I_{S}$ in Figure 17 suggests an important influence of both parameters, since it increases with $\mu_{r}$ and $C P^{-1}$. This behavior is more remarkable for lower values of $C P$, where FEM results deviate far from the sheath current estimated by Equation (26). It is also noticed that Equation (26) overestimates the induced current in the cases with no armor and stainless-steel armor. This is a consequence of the non-uniform current density distribution in the conductors due to skin and proximity effects, something not considered in Equation (26), where $I_{\mathcal{C}}$ is assumed to be applied in the center of the conductor $[10,33]$.

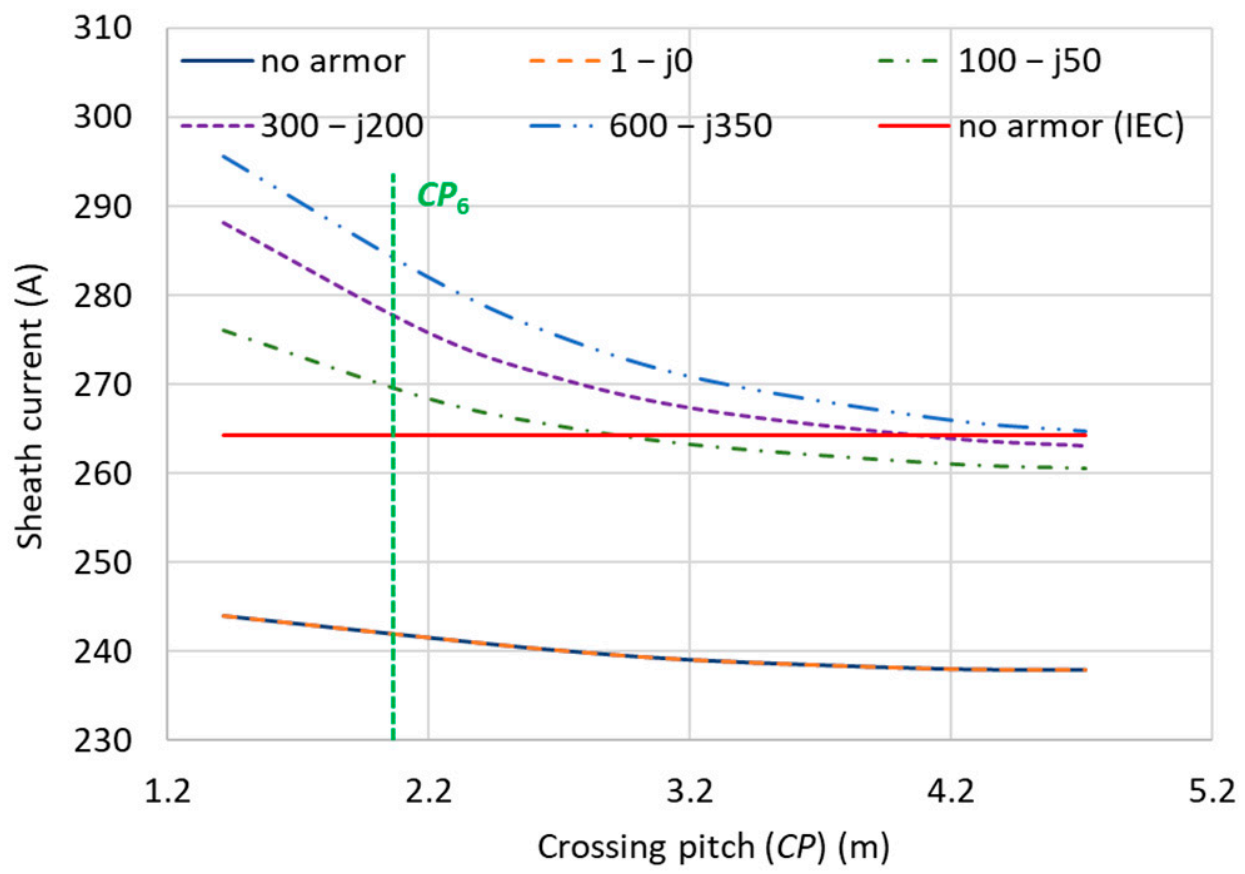

Figure 17. Variation of $I_{S}$ with $C P$ and $\mu_{r}$ for Cable $6(\mathrm{SB})$ (actual $C P_{6}$ of cable included as a reference).

Therefore, both $R_{s}$ and $X_{S}$ are also affected by all these aspects. Furthermore, the problem is even more complicated since the induced currents are non-uniformly distributed in the sheaths, as shown in Figure 18a for Cable 6 with $\mu_{r}=300-j 200$. In this sense, if $\gamma$ is employed for evaluating the changes in the loss distribution when the armor is considered (Figure 18b), an important increase is observed in the sheath losses in areas closer to the armor wires, where the electromagnetic coupling between both elements results in higher induced currents that contribute to increasing both eddy and circulating currents. 


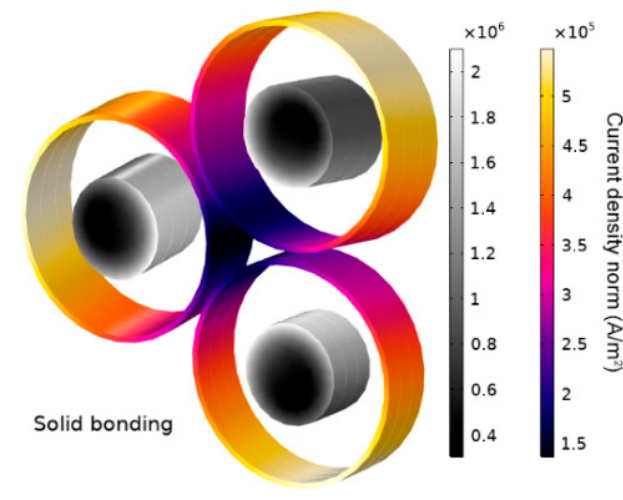

(a)

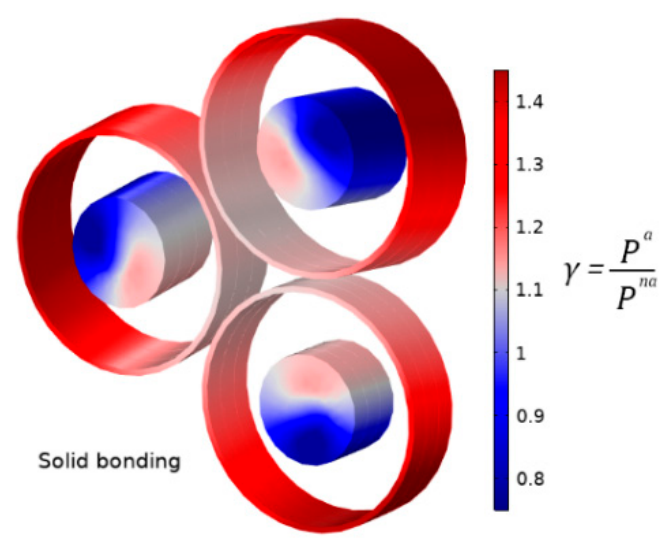

(b)

Figure 18. (a) Current density and (b) variation in the conductors and the sheath losses in Cable 6 when a magnetic armor of $\mu_{r}=300-j 200$ is installed (SB).

However, this does not necessarily lead to higher values of $R_{\mathcal{S}}$, as depicted in Figure 19a, where it is shown the influence of $\mu_{r}$ and CP on $R_{S}$ obtained by Equation (18) (DC resistance derived from Equation (24) is plotted as a reference). As can be seen, the impact of both $\mu_{r}$ and CP is weak, especially $\mu_{r}$, resulting in $R_{S}=R_{S}^{A C} \approx R_{S}^{D C}$, although the presence of the armor seems to lead to slightly lower values for $R_{S}$. This may be explained as the armor alleviating the proximity effect in the sheaths. Similar results are observed in Figure $19 b$, where the variation of $R_{S}$ with $\mu^{\prime}$ is represented for the 6 cables considered in this study, confirming the weak influence of $\mu_{r}$ on $R_{s}$.

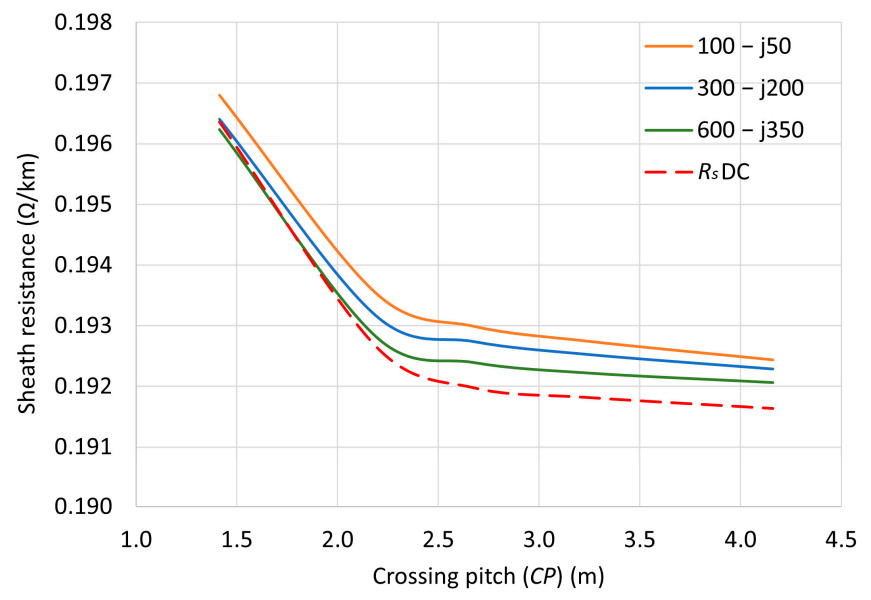

(a)

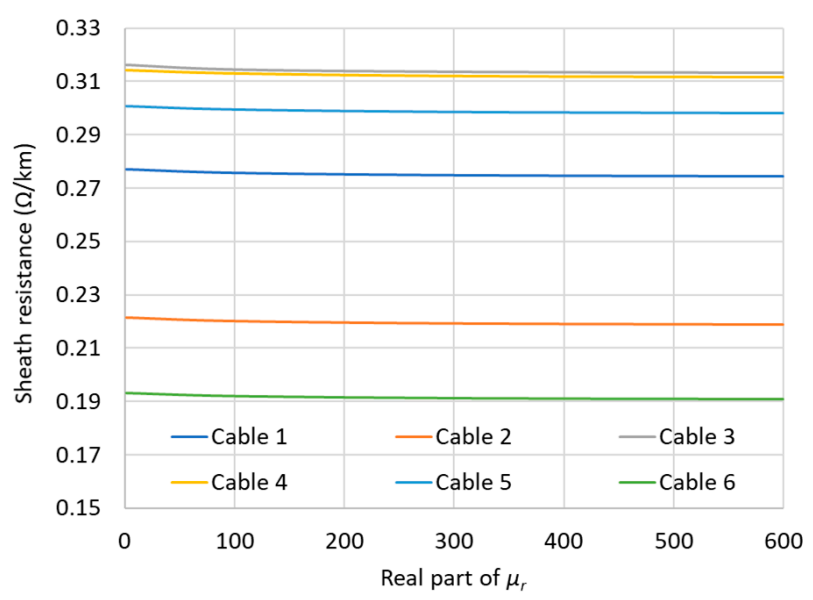

(b)

Figure 19. Variation of $R_{S}$ with (a) the $C P$ for Cable 6 for different values of $\mu_{r}$ and (b) the real part of $\mu_{r}$ for the 6 cables considered (SB).

Subsequently, since the armor has no important effects on $R_{s}$, the higher values observed for $I_{S}$ in Figure 17 suggest that $X_{S}$ is then largely affected. This underscores the need of improving the formulation for computing $X_{s}$, not only for obtaining $I_{s}$, but also for obtaining $\lambda^{\prime}{ }_{1}$, where both $C P$ and $\mu_{r}$ should play an important role. To this aim, the $3 \mathrm{D}$ ultra-shortened FEM model developed in this work is paving the way to refine Equation (26) in the future next. 


\subsection{Summary}

The main conclusions derived from all the analyses developed in this section are reflected in Figure 20, where the most relevant results, as well as the proposed solutions, are summarized for clarity. Their use for improving the experimental evaluation of $P_{a}$ is developed next.

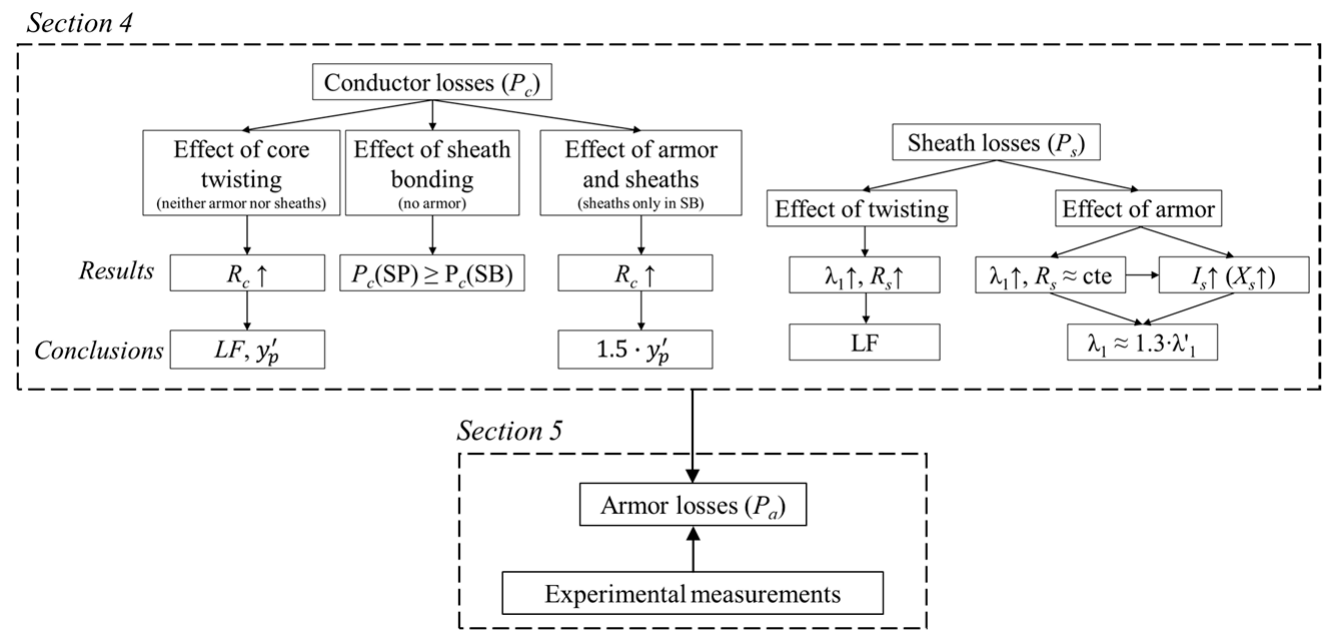

Figure 20. Main results and conclusions derived from Section 4 regarding $P_{c}$ and $P_{s}$, and their use as inputs for improving the experimental evaluation of $P_{a}$ proposed in Section 5.

\section{Estimation of Armor Losses}

As indicated previously, it's challenging to determine accurately the different components of the total losses in TCACs, especially through experimental measurements. In previous studies [26,28], different methods to reduce the measurement uncertainty were proposed, mainly based on measuring the total power dissipated in two three-core cables, one with armor and the other unarmored. During tests, different magnitudes are measured, such as the total power, the currents through the sheaths and conductors, as well as the DC resistance of metallic elements. Thus, $P_{a}$ is estimated by subtracting the power balance equations associated to both situations, where it is assumed that $R_{c}$ and $R_{S}$ are not influenced by the presence of the armor, hence having the same value in the two cables. The same applies for obtaining eddy losses in the sheaths by Equation (7). All this results in erroneous values for $P_{a}$, since they collect all deviations derived from these assumptions.

To overcome these problems, new and more accurate expressions for evaluating all these parameters would be required, as commented earlier. Meanwhile, the uncertainties derived from these assumptions may be lessened by applying the approaches proposed in previous section, like Equations (21) and (23) for computing $R_{c}$ in TCACs, since the impact of the armor and the cores twisting on this parameter is considered. Additionally, the relative weight of circulating losses in the total sheath losses found previously $\left(\lambda_{1} \approx 1.3 \lambda_{1}^{\prime}\right)$ may help. All this, combined with the available experimental measurements (DC resistance and current in conductors and sheaths), allow us to propose the following expressions for estimating the sheath and conductor losses:

$$
\begin{gathered}
\lambda_{1} \approx 1.3 \lambda_{1}^{\prime}=1.3 \frac{R_{s}(\theta)}{R_{c}(\theta)} \frac{I_{s, \text { meas }}^{2}}{I_{c, \text { meas }}^{2},} \\
P_{s}=\lambda_{1} P_{c}=1.3 \cdot 3 R_{s}(\theta) I_{s, \text { meas }}^{2}, \\
P_{c}=3 R_{c}(\theta) I_{c, \text { meas }}^{2},
\end{gathered}
$$


where $I_{c, \text { meas }}$ and $I_{s, \text { meas }}$ represent the currents measured in conductors and sheaths, respectively. This way, the armor losses can be derived from the total power dissipated during the test $\left(P_{\text {total }}^{\text {meas }}\right)$ as

$$
P_{a}=P_{\text {total }}^{\text {meas }}-P_{c}-P_{s}
$$

Even though the procedure includes experimental measurements, we have applied equivalent virtual tests in 750 simulations performed by means of the 3D-FEM model presented in this work, obtaining for each test the "virtual measurements" previously cited: $R_{c}^{D C}, R_{S}^{D C}, I_{c, \text { meas }}, I_{s, \text { meas }}$ and $P_{\text {total }}^{\text {meas }}$. Regarding the 750 simulations, they consist in numerous situations obtained by modifying some of the main properties of a group of more than 50 TCACs ranging from $10 \mathrm{kV}, 35 \mathrm{~mm}^{2}$ to $275 \mathrm{kV}, 2000 \mathrm{~mm}^{2}$. Changes in the number and thickness of the armor wires, the phase and armor lay length, as well as the sheath thickness and $\mu_{r}=\mu^{\prime}-j \mu^{\prime \prime}$ were applied to increase the number of cases. Further, copper and aluminum conductors were considered. Finally, several test cases include the increase in the electrical resistivity due to the temperature rise in the power cable. Only SB configuration has been considered in all the cases. Table 4 summarizes the range of the main numerical parameters employed in this study.

Table 4. Maximum and minimum values of the main numerical parameters of all TCACs simulated.

\begin{tabular}{ccc}
\hline & Min & Max \\
\hline Cross section $\left(\mathrm{mm}^{2}\right)$ & 35 & 2000 \\
Voltage $(\mathrm{kV})$ & 10 & 275 \\
Sheath diameter $(\mathrm{mm})$ & 17 & 121 \\
Sheath thickness $(\mathrm{mm})$ & 1 & 4.5 \\
Conductor lay length $(\mathrm{m})$ & 0.5 & 5 \\
Armor lay length $(\mathrm{m})$ & 0.5 & 5 \\
$C P(\mathrm{~m})$ & 0.3 & 2.2 \\
Armor wire diameter $(\mathrm{mm})$ & 3.15 & 7 \\
$\rho_{c}(\Omega \cdot \mathrm{m})$ & $1.17 \cdot 10^{-8}$ & $3.53 \cdot 10^{-8}$ \\
$\rho_{s}(\Omega \cdot \mathrm{m})$ & $1.71 \cdot 10^{-7}$ & $3.21 \cdot 10^{-7}$ \\
$\rho_{a}(\Omega \cdot \mathrm{m})$ & $1.7 \cdot 10^{-7}$ & $2.07 \cdot 10^{-7}$ \\
$\mu^{\prime}$ & 50 & 600 \\
$\mu^{\prime \prime}$ & 0 & 350 \\
\hline
\end{tabular}

Eventually, the values of $R_{c}$ and $R_{s}$ are computed by means of Equations (21)-(25). Having all these data, $P_{c}, P_{s}$ and $P_{a}$ are obtained by means of Equations (28)-(30), and then compared to those obtained exclusively with FEM simulations, resulting in the error represented in Figure 21a. As can be easily observed, it is quite remarkable the good agreement shown between the estimated values and the FEM results, especially for $P_{\mathcal{C}}$ and $P_{S}$, since the maximum error barely exceeds $5 \%$ in just a few cases. In addition, a noticeable improvement is achieved in $P_{a}$, with relative errors lower than $20 \%$ in most of the cases, improving the results previously observed in [16] where differences above $60 \%$ were usually obtained by the experimental procedures of $[26,28]$.

Be aware that, in this case, a relative error below $20 \%$ is in practice negligible due to the small absolute value of $P_{a}$, as can be observed in Figure 21b, where the result derived by FEM and [5] are represented, being the latter substantially overestimated. Precisely, the great differences between the absolute values of $P_{a}$ and $P_{c}$, together with the highest value of $\mu_{r}$, are the cause of the highest errors observed in Figure 21a. In those situations, Equation (23) underestimates $P_{c}$ in about $1-2 \%$. This results in $1-3 \mathrm{~W} / \mathrm{m}$ of deviation that is later assigned to $P_{a}$ by Equation (30). However, this quantity is of the same order of magnitude of the actual value of $P_{a}$ obtained in those cases, thus causing the highest errors. Even so, less than $4 \%$ of the 750 cases analyzed in this work have a relative error greater than $20 \%$ in $P_{a}$. 


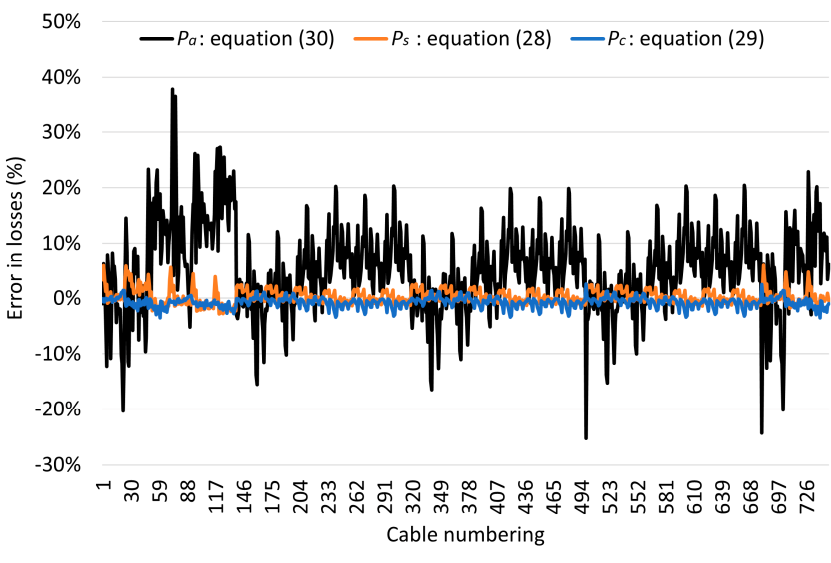

(a)

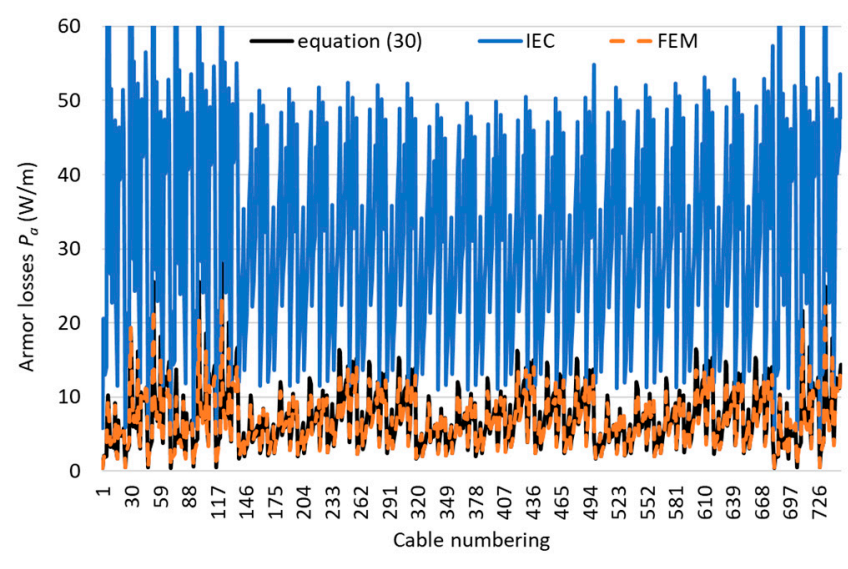

(b)

Figure 21. (a) Error, relative to FEM simulations, in the estimation of conductor, sheath and armor losses, and (b) armor losses derived from the estimation, FEM and [5] (SB).

It is true that the application of the proposed approach in a laboratory setup will not be as accurate, mainly due to measurement and calibration errors. In any case, the approach presented in this work allows to avoid the erroneous assumptions considered in previous procedures $[26,28]$, hence increasing the accuracy of the estimations. Furthermore, it also avoids the need of taking measurements in two cables, although this can be interesting for minimizing errors.

\section{Conclusions}

This paper analyses the problem of properly quantifying and allocating the losses in the three metallic components (conductors, sheaths and magnetic armor) of TCACs commonly employed in submarine energy transmission. To this aim, an ultra-shortened and improved 3D-FEM model is proposed to perform fast and accurate analyses in 6 real cables. The influence of different aspects, such as the sheath bonding, conductor twisting, and the presence of a magnetic armor on the conductor and sheath losses is illustrated and quantified thanks to the new features of the proposed model.

From this analysis, it is concluded that both sheath and conductor losses increase with the cores and armor twisting, as well as with the magnetic permeability of the armor. Moreover, thanks to the new features implemented in the ultra-shortened 3D-FEM model, changes in the loss allocation can be visualized, concluding that the increase observed in the conductor losses may be identified as an increase in the proximity effect inside the conductor. On the other hand, the results also show how a magnetic armor leads to higher sheath currents, being this behavior a consequence of an increase in the sheath reactance.

As a result, different corrections are proposed for improving the estimation of the conductor and sheath losses, being applied in a new and simplified procedure suggested for the experimental evaluation of the armor losses, where the 3D-FEM model is employed as a "virtual laboratory". The procedure has been tested with more than 700 cable configurations showing excellent results, with differences below $5 \%$ in the sheath and conductor losses when compared to FEM results. Further, a good agreement is observed in the armor losses, with differences below $20 \%$, substantially reducing the errors observed in previous experimental procedures.

Even though the procedure includes experimental measurements, the benefits of using FEM-based virtual tests are also remarked, such as reducing noticeably the experimental burden, and improving the knowledge of all the phenomena involved in this type of cable. In this sense, the proposed 3D-FEM model is currently employed in finding new and more accurate expressions, not only for evaluating the cable losses, but also for obtaining its electrical parameters, being all this in line with the remarkable efforts of on-going CIGRÉ working groups B1.64 and B1.56. 
Author Contributions: Formal analysis, J.C.d.-P.-L. and P.C.-R.; Investigation, P.C.-R.; Methodology, J.C.d.-P.-L. Validation, J.C.d.-P.-L. and P.C.-R.; Writing—original draft, J.C.d.-P.-L. Writing-review \& editing, P.C.-R. All authors have read and agreed to the published version of the manuscript.

Funding: This research was funded by the FEDER/Ministerio de Ciencia e Innovación-Agencia Estatal de Investigación under the project ENE2017-89669-R and by the Universidad de Sevilla (VI PPIT-US) under grant 2018/00000740.

Institutional Review Board Statement: Not applicable.

Informed Consent Statement: Not applicable.

Data Availability Statement: Not applicable.

Conflicts of Interest: The authors declare no conflict of interest.

\section{References}

1. Worzyk, T. Submarine Power Cables. Design, Installation, Repair, Environmental Aspects; Springer Science \& Business: Berlin, Germany, 2009; ISBN 978-3-642-01269-3.

2. CIGRE. TB 815 Update of Service Experience of HV Underground and Submarine Cable Systems; CIGRE: Paris, France, 2020.

3. Bremnes, J.J.; Evenset, G.; Stolan, B. Power loss and inductance of steel armored multi-core cables: Comparison of IEC values with “2.5D” FEA results and measurements. In Proceedings of the CIGRÉ Session, Paris, France, 22-27 August 2010.

4. Hatlo, M.; Bremnes, J.J. Current dependent armour loss in three-core cables: Comparison of FEA results and measurements. In Proceedings of the CIGRÉ Session, Paris, France, 24-29 August 2014.

5. IEC. 60287-1-1 Electric Cables-Calculation of the Current Rating-Part 1-1: Current Rating Equations (100 \% load factor) and Calculation of Losses-General; IEC: Geneva, Switzerland, 2014.

6. Hatlo, M.; Olsen, E.; Stølan, R. Accurate analytic formula for calculation of losses in three-core submarine cables. In Proceedings of the 9th International Conference on Insulated Power Cables, Versailles, France, 21-25 June 2015.

7. Goddard, K.F.; Pilgrim, J.A.; Chippendale, R.D.; Lewin, P.L. Induced losses in three-core SL-type high-voltage cables. IEEE Trans. Power Deliv. 2015, 30, 1505-1513. [CrossRef]

8. Gustavsen, B.; Hoyer-Hansen, M.; Triverio, P.; Patel, U.R. Inclusion of Wire Twisting Effects in Cable Impedance Calculations. IEEE Trans. Power Deliv. 2016, 31, 2520-2529. [CrossRef]

9. Giussani, L.; Di Rienzo, L.; Bechis, M.; De Falco, C. Computation of Armor Losses in AC Submarine Cables. IEEE Trans. Power Deliv. 2020. [CrossRef]

10. da Silva, F.M.F.; Ebdrup, T.; Bak, C.L.; Jensen, C.F. Understanding losses in three core armoured submarine cables. In Proceedings of the CIGRÉ Session, Paris, France, 21-26 August 2016.

11. Expethit, A.; Sørensen, S.B.; Arentsen, M.T.; Pedersen, M.V.; Sørensen, D.A.; da Silva, F.M.F. MVAC Submarine cable, magnetic fields measurements and analysis. In Proceedings of the 2017 52nd International Universities' Power Engineering Conference (UPEC), Heraklion, Greece, 28-31 August 2017. [CrossRef]

12. Sturm, S.; Paulus, J. Estimating the losses in three-core submarine power cables using 2D and 3D FEA simulations. In Proceedings of the 9th International Conference on Insulated Power Cables, Versailles, France, 21-25 June 2015.

13. Benato, R.; Sessa, S.D.; Forzan, M.; Marelli, M.; Pietribiasi, D. Core laying pitch-long 3D finite element model of an AC three-core armoured submarine cable with a length of 3 meters. Electr. Power Syst. Res. 2017, 150, 137-143. [CrossRef]

14. del-Pino-López, J.; Hatlo, M.; Cruz-Romero, P. On Simplified 3D Finite Element Simulations of Three-Core Armored Power Cables. Energies 2018, 11, 3081. [CrossRef]

15. Bompou, E.; Mijatovic, N.; Traholt, C.; Willen, D.W.A.; Thidemann, C. Loss in Steel Armour Wires for Submarine Power Cables. In Proceedings of the 2018 IEEE Power \& Energy Society General Meeting (PESGM), Portland, OR, USA, 5-10 August 2018. [CrossRef]

16. del-Pino-López, J.C.; Cruz-Romero, P.; Sanchez-Díaz, L.C. Loss Allocation in Submarine Armored Three-core HVAC Power Cables. In Proceedings of the 2020 IEEE International Conference on Environment and Electrical Engineering and 2020 IEEE Industrial and Commercial Power Systems Europe (EEEIC/I and CPS Europe), Madrid, Spain, 9-12 June 2020. [CrossRef]

17. Benato, R.; Dambone Sessa, S. A New Multiconductor Cell Three-Dimension Matrix based Analysis Applied to a Three-Core Armoured Cable. IEEE Trans. Power Deliv. 2017, 33, 1636-1646. [CrossRef]

18. Benato, R.; Sessa, S.D. Computation of power losses in HV submarine three-core armoured cables: A 3D multiconductor cell analysis along with subdivision technique. In Proceedings of the CIGRE Session, Paris, France, 26-31 August 2018.

19. Benato, R.; Dambone Sessa, S.; Guizzo, L.; Palone, F. Capri-Torre Annunziata 150 kV Three-core armoured submarine cable: A first 3D multiconductor cell analysis confirmation. In Proceedings of the AEIT International Annual Conference, Bari, Italy, 3-5 October 2018. [CrossRef]

20. Benato, R.; Sessa, S.D.; Forzan, M. Experimental Validation of 3-Dimension Multiconductor Cell Analysis by a $30 \mathrm{~km}$ Submarine Three-Core Armoured Cable. IEEE Trans. Power Deliv. 2018, 33, 2910-2919. [CrossRef] 
21. Stølan, R.; Hatlo, M. Armour Loss in Three Core Submarine Cables-Measurements of Cable Impedance and Armour Wire Permeability. In Proceedings of the CIGRE Session, Paris, France, 26-31 August 2018.

22. Arentsen, M.T.; Pedersen, M.V.; Sjilrensen, S.B.; Arentsen, M.T.; Expethit, A.; Pedersen, M.V.; Sorensen, S.B. MVAC Submarine Cable, Impedance Measurements and Analysis. In Proceedings of the 2017 52nd International Universities' Power Engineering Conference (UPEC), Heraklion, Greece, 28-31 August 2017. [CrossRef]

23. Lee, J.M.; Park, D.J.; Jeong, G.Y. Measurements and FEA Results of Steel Armour Losses in Three-core Submarine XLPE. In Proceedings of the CIGRÉ, Paris, France, 21-26 August 2016.

24. Ebdrup, T.; da Silva, F.M.F.; Bak, C.L.; Jensen, C.F. Comparison of losses in an armoured and unarmoured three phase cable. In Proceedings of the 9th International Conference on Insulated Power Cables, Versailles, France, 21-25 June 2015.

25. Maioli, P.; Bechis, M.; Dell'anna, G. AC resistance of submarine cables. In Proceedings of the 9th International Conference on Insulated Power Cables, Versailles, France, 21-25 June 2015.

26. Palmgren, D. Armour loss in three-core submarine XLPE cables. In Proceedings of the 8th International Conference on Insulated Power Cables, Versailles, France, 19-23 June 2011.

27. Pilgrim, J.A.; Goddard, K.F. Accurate Measurement of Losses in Three Core Armoured Cables. In Proceedings of the 10th International Conference on Insulated Power Cables, Versailles, France, 23-27 June 2019.

28. Frelin, W.; Moreau, C.; Willen, D.; Thidemann, C.; De Robien, G.; Boudinet, N. Measurements of losses on three core submarine power cables. In Proceedings of the 9th International Conference on Insulated Power Cables, Versailles, France, 21-25 June 2015.

29. De Wild, F.; Anders, G.J.; Kamara, W.; Canada, C. Overview of CIGRÉ WG B1.56 regarding the verification of cable current ratings. In Proceedings of the 10th International Conference on Insulated Power Cables, Versailles, France, $23-27$ June 2019.

30. Chatzipetros, D. Improved Current Rating Methods of Subsea HVAC Cable Systems for Renewable Energy Applications. Ph.D. Thesis, University of Southampton, Southampton, UK, October 2019.

31. Sturm, S.; Küchler, A.; Paulus, J.; Stølan, R.; Berger, F. 3D-FEM modelling of losses in armoured submarine power cables and comparison with measurements. In Proceedings of the CIGRE e-Session, Paris, France, 24 August-3 September 2020.

32. Barrett, J.S.; Anders, G.J. Circulating current and hysteresis losses in screens, sheaths and armour of electric power cablesmathematical models and comparison with IEC Standard 287. IEE Proc.-Sci. Meas. Technol. 1997, 144, 101-110. [CrossRef]

33. Chatzipetros, D.; Pilgrim, J.A. Impact of Proximity Effects on Sheath Losses in Trefoil Cable Arrangements. IEEE Trans. Power Deliv. 2019, 35, 455-463. [CrossRef]

34. Chatzipetros, D.; Pilgrim, J.A. Induced Losses in Non-Magnetically Armoured HVAC Windfarm Export Cables. In Proceedings of the 2018 IEEE International Conference on High Voltage Engineering and Application (ICHVE), Athens, Greece, 10-13 September 2018. [CrossRef]

35. COMSOL Multiphysics ${ }^{\circledR}$ v. 5.5. COMSOL AB.; Stockholm; Sweden. Available online: http:/ /www.comsol.com (accessed on 30 March 2021).

36. Del-Pino-Lopez, J.C.; Cruz-Romero, P. Hybrid 2D/3D fully coupled electrothermal model for three-core submarine armored cables. In Proceedings of the Comsol Conference Europe, Online, 14-15 October 2020.

37. Del-Pino-López, J.C.; Cruz-Romero, P. A 3D parametric thermal analysis of submarine three-core power cables. In Proceedings of the Renewable Energy and Power Quality Journal, Granada, Spain, 2-4 September 2020.

38. CIGRE. TB 797-Sheath Bonding Systems of ac Transmission Cables; CIGRE: Paris, France, 2020. 